\title{
Laminar flow downregulates Notch activity to promote lymphatic sprouting
}

\author{
Dongwon Choi, ${ }^{1}$ Eunkyung Park, ${ }^{1}$ Eunson Jung, ${ }^{1}$ Young Jin Seong, ${ }^{1}$ Jaehyuk Yoo, ${ }^{1}$ Esak Lee, ${ }^{2}$ Mingu Hong, ${ }^{1}$ Sunju Lee, ${ }^{1}$ \\ Hiroaki Ishida, ${ }^{3}$ James Burford, ${ }^{4}$ Janos Peti-Peterdi, ${ }^{4}$ Ralf H. Adams, ${ }^{5}$ Sonal Srikanth, ${ }^{6}$ Yousang Gwack, ${ }^{6}$ \\ Christopher S. Chen, ${ }^{2}$ Hans J. Vogel, ${ }^{3}$ Chester J. Koh, ${ }^{7}$ Alex K. Wong, ${ }^{1}$ and Young-Kwon Hong ${ }^{1}$ \\ 'Division of Plastic and Reconstructive Surgery, Department of Surgery, Norris Comprehensive Cancer Center, Keck School of Medicine, University of Southern California, Los Angeles, California, USA. \\ 2Department of Biomedical Engineering and the Biological Design Center, Boston University; and Wyss Institute for Biologically Inspired Engineering, Harvard University, Boston, Massachusetts, USA. \\ ${ }^{3}$ Department of Biological Sciences, University of Calgary, Calgary, Alberta, Canada. ${ }^{4}$ Department of Physiology and Biophysics, Zilkha Neurogenetic Institute, Keck School of Medicine, University of \\ Southern California, Los Angeles, California, USA. ${ }^{5}$ Max Planck Institute for Molecular Biomedicine, Department of Tissue Morphogenesis, and University of Münster, Faculty of Medicine, Münster, Cermany. \\ ${ }^{6}$ Department of Physiology, David Geffen School of Medicine at UCLA, Los Angeles, California, USA. Division of Pediatric Urology, Texas Children's Hospital, Baylor College of Medicine, Houston, Texas, USA.
}

\begin{abstract}
The major function of the lymphatic system is to drain interstitial fluid from tissue. Functional drainage causes increased fluid flow that triggers lymphatic expansion, which is conceptually similar to hypoxia-triggered angiogenesis. Here, we have identified a mechanotransduction pathway that translates laminar flow-induced shear stress to activation of lymphatic sprouting. While low-rate laminar flow commonly induces the classic shear stress responses in blood endothelial cells and lymphatic endothelial cells (LECs), only LECs display reduced Notch activity and increased sprouting capacity. In response to flow, the plasma membrane calcium channel ORAl1 mediates calcium influx in LECs and activates calmodulin to facilitate a physical interaction between Krüppel-like factor 2 (KLF2), the major regulator of shear responses, and PROX1, the master regulator of lymphatic development. The PROX1/KLF2 complex upregulates the expression of DTX1 and DTX3L. DTX1 and DTX3L, functioning as a heterodimeric Notch E3 ligase, concertedly downregulate NOTCH1 activity and enhance lymphatic sprouting. Notably, overexpression of the calcium reporter GCaMP3 unexpectedly inhibited lymphatic sprouting, presumably by disturbing calcium signaling. Endothelial-specific knockouts of Orai1 and KIf2 also markedly impaired lymphatic sprouting Moreover, Dtx3l loss of function led to defective lymphatic sprouting, while $D t x 3 /$ gain of function rescued impaired sprouting in Orai1 $\mathrm{KO}$ embryos. Together, the data reveal a molecular mechanism underlying laminar flow-induced lymphatic sprouting.
\end{abstract}

\section{Introduction}

The lymphatic system consists of lymphatic networks and lymphoid organs (1). Unlike the blood vascular system, where the fluid (blood) leaves and returns to the same organ (the heart), lymphatic vessels start from the tissue interstitium as capillary lymphatics, become the collectors, and eventually end at the venous connection as the thoracic duct, the biggest-caliber lymphatic vessel. While capillary lymphatics are composed of a single layer of overlapping lymphatic endothelial cells (LECs) and are devoid of mural cells and continuous basement membrane, collecting lymphatic vessels are equipped with luminal valves, as well as mural cells and basement membrane. Whereas LECs in lymphatic capillaries are expected to experience a basal-to-apical interstitial flow, followed by unidirectional laminar flow to downstream compartments, the cells in the collecting lymphatics are more likely exposed to oscillatory flow.

Blood vessels carry a relatively constant volume of blood and remain distended at normal steady state. Lymphatic capillaries, however, remain collapsed until a significant volume of intersti-

Conflict of interest: The authors have declared that no conflict of interest exists

Submitted: March 9, 2016; Accepted: January 11, 2017.

Reference information: J Clin Invest. 2017;127(4):1225-1240.

https://doi.org/10.1172/JCl87442. tial fluid flows into the lumen. As tissue fluid drainage is a primary function of lymphatic vessels, fluid flow was hypothesized to serve as an important nonbiological lymphangiogenic stimulus (2). Indeed, the initial pioneering studies using in vivo models showed that interstitial fluid flow caused by functional drainage serves as a critical morphogenic mediator of lymphatic vessel organization by controlling LEC migration, VEGF-C expression, and lymphatic capillary network formation (3-5). An increase in embryonic fluid drainage was previously found to coincide with the initial lymphatic development and serve as a signal for embryonic lymphatic expansion (6). Lymph drainage and flow were also shown to regulate collecting lymphatic vessel maturation as well as luminal valve formation and development in vivo $(7,8)$. A recent study using a $3 \mathrm{D}$ in vitro biomimetic model showed that interstitial flow alone is sufficient to activate lymphatic sprouting, and that this mechanical force synergizes with biological stimuli to enhance lymphatic growth (9). Therefore, flow-induced mechanical signals, together with biological stimuli, seem to play critical roles in lymphatic growth, expansion, maturation, valve formation, and remodeling (10).

Studies have shown that steady laminar flow at a rate equivalent to blood flow (greater than $10 \mathrm{dyn} / \mathrm{cm}^{2}$ ) causes a variety of responses in blood vessel endothelial cells (BECs), including elongated cell morphology, cell proliferation arrest, increased 
calcium entry, Notch activation, and upregulation of Krüppel-like factor 2 (KLF2), the master regulator of the shear stress response (11-17). Moreover, fluid shear stress can promote endothelial differentiation of bone marrow-derived progenitor cells and embryonic stem cells in vitro (16). Similarly, blood flow reprograms lymphatic vessels to blood vessels in mice (18). Thus, it is clear that fluid flow has a substantial effect on establishment and maintenance of the blood vascular system. In comparison, the molecular basis of functional drainage-induced lymphatic expansion remains undefined. In this study, we aimed to gain a mechanistic understanding of how the flow functions as a growth stimulus for lymphatic vessels during development. Our data show that laminar flow activates ORAI1, a major component of the calcium release-activated calcium (CRAC) channel, and results in calcium influx in LECs. Increased intracellular calcium, in turn, activates calmodulin $(\mathrm{CaM})$ to promote a complex formation between a key shear stress regulator, KLF2, and the master regulator of lymphatic development, PROX1. The resulting KLF2/PROX1/ $\mathrm{Ca}^{2+}-\mathrm{CaM}$ protein complex upregulates a heterodimeric Notch E3 ligase, encoded by Dtx1 (deltex E3 ubiquitin ligase 1) and Dtx3l (deltex E3 ubiquitin ligase 3L), which activates lymphatic sprouting through suppression of Notch activity. Together, our findings uncover a molecular mechanism underlying the laminar flowinduced lymphatic expansion.

\section{Results}

Laminar flow selectively suppresses NOTCH activity in LECs and increases lymphatic sprouting. We aimed to study the impact of lowrate laminar flow generated from functional lymphatic drainage on lymphatic development. Currently, the shear force levels in the developing embryonic lymphatic network are unknown. Thus, we applied various levels of laminar shear force on cultured LECs and found that steady laminar flow at approximately $2 \mathrm{dyn} / \mathrm{cm}^{2}$ or higher clearly and consistently generated the classic endothelial shear stress responses, such as elongated cell morphology, upregulation of KLF2 and endothelial NOS, and calcium influx (Figure 1A; Supplemental Figure 1, A and B, and Supplemental Figure 2, $A$ and B; supplemental material available online with this article; doi:10.1172/JCI87442DS1). Unexpectedly, the laminar flow at this shear force caused downregulation of the NOTCH target genes NRARP, HEY1, and HEY2 in LECs (Figure 1B). BECs, in comparison, did not show the same regulation of the Notch pathway (Supplemental Figure 1C), possibly because the applied shear force $\left(\sim 2 \mathrm{dyn} / \mathrm{cm}^{2}\right)$ was much lower than the physiological levels in blood vessels (10-20 dyn/ $\left.\mathrm{cm}^{2}\right)$. Moreover, whereas the mRNA level of NOTCH1 was not altered (Supplemental Figure 1D), the protein level of the NOTCH1 intracellular domain (NICD1) was significantly reduced in LECs, but not in BECs, by the laminar flow (Figure 1C and Supplemental Figure 1E). In comparison, NICD4 protein level was not changed in either cell type by laminar flow (Supplemental Figure 1, F and G). Consistent with these findings, a Notch activity reporter assay using an RBP-JK-responsive luciferase vector confirmed that the steady laminar flow decreased Notch activity in LECs (Figure 1D). Notably, the PROX1 protein level was not changed by this experimental condition (Supplemental Figure $1 \mathrm{H})$, although a higher flow rate $\left(20 \mathrm{dyn} / \mathrm{cm}^{2}\right)$ downregulated PROX1 with loss of the LEC identity (18).
Because Notch activity has been shown to be a key regulator of lymphatic sprouting (19-23), we next evaluated the effect of the low-rate laminar flow on the in vitro sprouting potential of LECs by spheroid-based sprouting and microfluidic vascular mimetic assays (24-27). In spheroid-based sprouting assays (24, 25), flow-exposed LECs displayed increased sprout-forming capacity, whereas the same treatment in BECs did not show the same phenotype (Figure 1E). We next created a 3D vascular mimetic model by lining microfluidic channels with single-donor-derived BECs and LECs and subsequently subjected them to steady intraluminal laminar flow $(26,27)$. Consistent with the spheroid-based sprouting assays, laminar flow strongly enhanced LEC sprouting, but did not induce the same response in BECs (Figure $1 \mathrm{~F}$ and Supplemental Figure 1I). These data show that although LECs and BECs similarly exhibit the conventional shear stress responses to the low-rate steady laminar flow, only LECs increase their sprouting capability by suppressing Notch.

Calcium influx through ORAI1 is essential for the laminar flowinduced suppression of Notch activity and enhanced sprouting in LECs. As intracellular calcium mobilization is a hallmark immediate response to shear stress in endothelial cells, we next studied the dynamics of laminar flow-induced calcium influx in LECs. The calcium signal was activated in LECs within the first minute of the onset of laminar flow, after which it gradually decreased despite the continuous presence of laminar flow (Figure 2, A and B), consistent with a previous study (28). Since the calcium channel responsible for the flow-induced intracellular calcium increase has not been identified in LECs, we searched for the key calcium channel protein that may control the downstream shear stress responses in LECs, particularly Notch downregulation. Our search found that inhibition of store-operated calcium entry (SOCE) (29) with low concentrations of SKF-96365 (a nonspecific inhibitor of SOCE) could efficiently block the laminar flow-induced calcium mobilization in LECs (Figure 2, A and B). More specifically, when we knocked down ORAI1, a pore subunit of the CRAC channel that is involved in the SOCE process (30), the flow-mediated reduction of NICD1 as well as the increased calcium influx in LECs was completely abrogated (Figure 2C and Supplemental Figure 2), suggesting that ORAI1 is responsible for the laminar flow-induced calcium mobilization and NOTCH1 downregulation in LECs. Moreover, ORAI1 knockdown also significantly reversed the flowinduced downregulation of Notch pathway genes in LECs, but did not yield any effects in BECs (Figure 2D). In addition, ORAI1 knockdown abolished the in vitro sprouting capacity of LECs stimulated by laminar flow, while not altering BEC sprouting (Figure 2E). These data imply that ORAI1, responsible for the laminar flow-induced calcium entry, is essential for the NOTCH1 suppression and enhanced sprouting in LECs.

ORAI1 function is required for lymphatic sprouting during development. We next investigated the vascular phenotypes in Orai1 KO mice (31). Conventional Orai1 KO embryos and rarely surviving postnatal mice displayed abnormally enlarged lymphatic vessels with reduced number of sprouts and branches in their back skins and ears, respectively (Figure 3, A and B, and Supplemental Figure 3). Morphometric analyses revealed that Orai1 knockout caused defective lymphatic development, characterized by reduced number $(\sim 50 \%)$ of lymphatic branching points and increased distance 


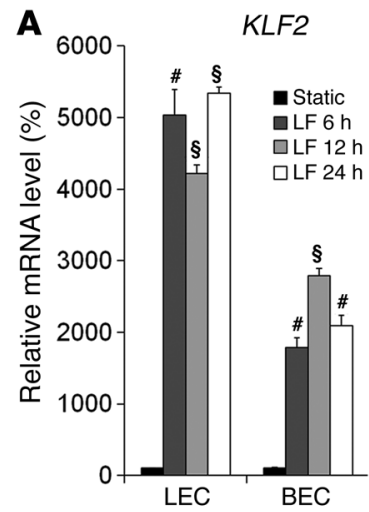

B

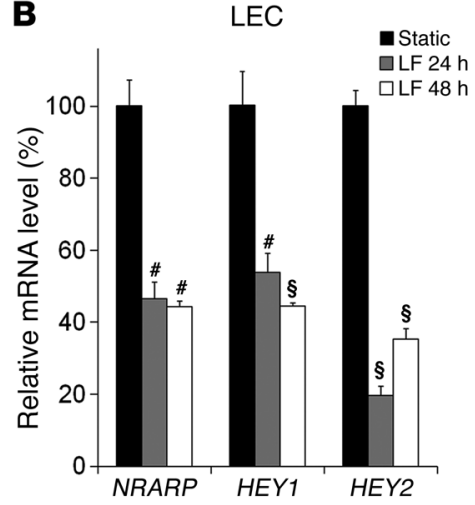

C

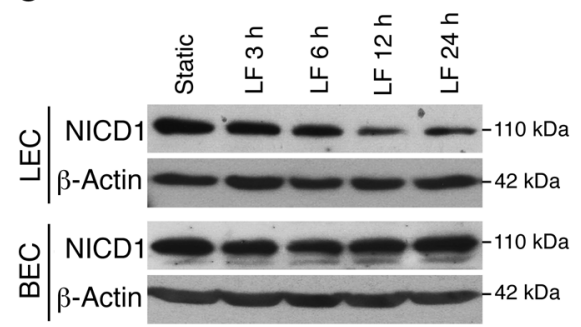

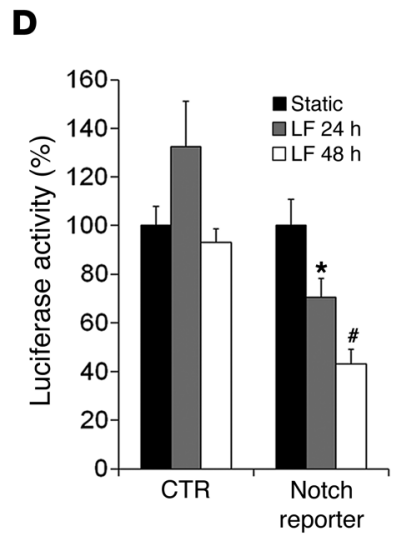

$\mathbf{F}$
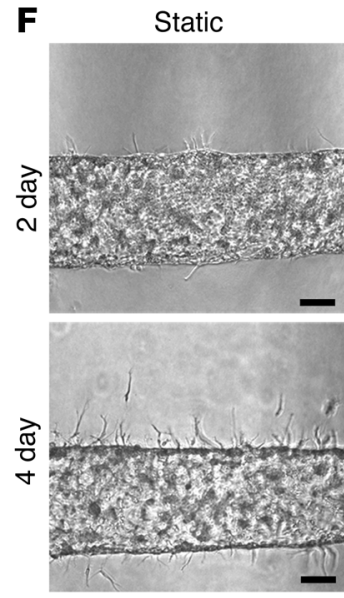

E
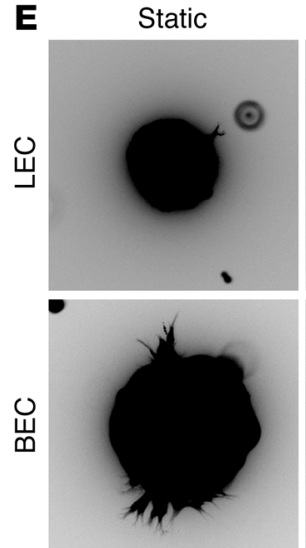
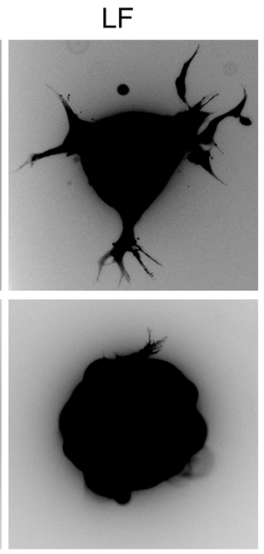

LF

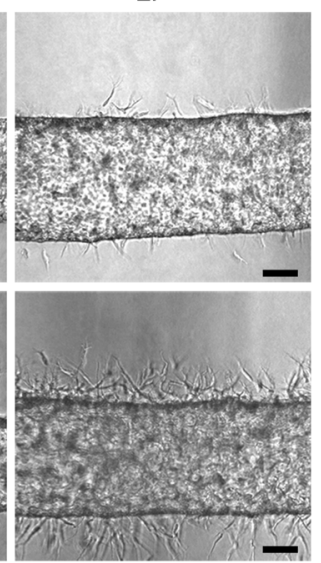

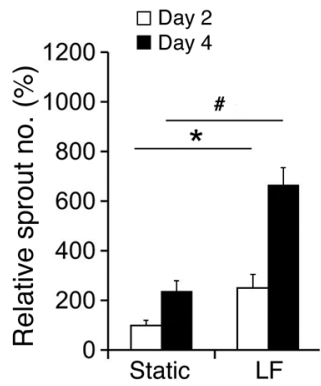

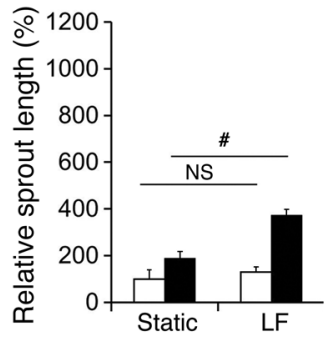

Figure 1. Laminar flow selectively suppresses NOTCH1 activity in LECs. (A and B) Real-time quantitative reverse transcription PCR (qRT-PCR) data showing the mRNA levels of KLF2 in LECs and BECs (A) and those of Notch target genes in LECs (B) in response to steady laminar flow $\left(2 \mathrm{dyn} / \mathrm{cm}^{2}\right)$. Expression of the Notch target genes in BECs by laminar flow is shown in Supplemental Figure 1C. (C) Protein levels of NICD1 in LECs and BECs in response to laminar flow $\left(2 \mathrm{dyn} / \mathrm{cm}^{2}\right)$. The intensity of the Western blotting bands is quantitated in Supplemental Figure 1E. A monoclonal anti-NOTCH1 antibody that specifically detects the cleaved form of NOTCH1 at Val1744 was used. Blots presented are derived from replicate samples run on parallel gels. (D) Luciferase assay showing the flow-mediated suppression of Notch activity in LECs. LECs were transfected with a Notch activity reporter (pGa981-6) (60) and exposed to laminar flow $\left(2 \mathrm{dyn} / \mathrm{cm}^{2}\right)$ for 24 or 48 hours before measurement of luciferase activity. (E) Spheroid-based sprouting assays. Cells were exposed or not exposed to laminar flow ( $2 \mathrm{dyn} / \mathrm{cm}^{2}$ ) for 24 hours, stained with a CellTracker dye, aggregated in methylcellulose polymers, and then embedded in Matrigel. After 24 hours, images of more than 20 spheroids were taken for analyses, and relative sprout numbers were quantified in the graph. (F) Biomimetic sprouting assay. Intraluminal laminar flow $\left(5 \mathrm{dyn} / \mathrm{cm}^{2}\right)$ was applied onto a layer of LECs lining the inner wall of the vascular mimetic channels made in collagen gel. Scale bars: $100 \mu \mathrm{m}$. Relative sprout length and number were graphed. Each experiment was independently performed at least 3 times with consistent results. Data are expressed as SEM and SD from 1 representative data set. ${ }^{*} P<0.05$; ${ }^{\#} P<0.01$; $\$ P<0.001 ; t$ test. 
A
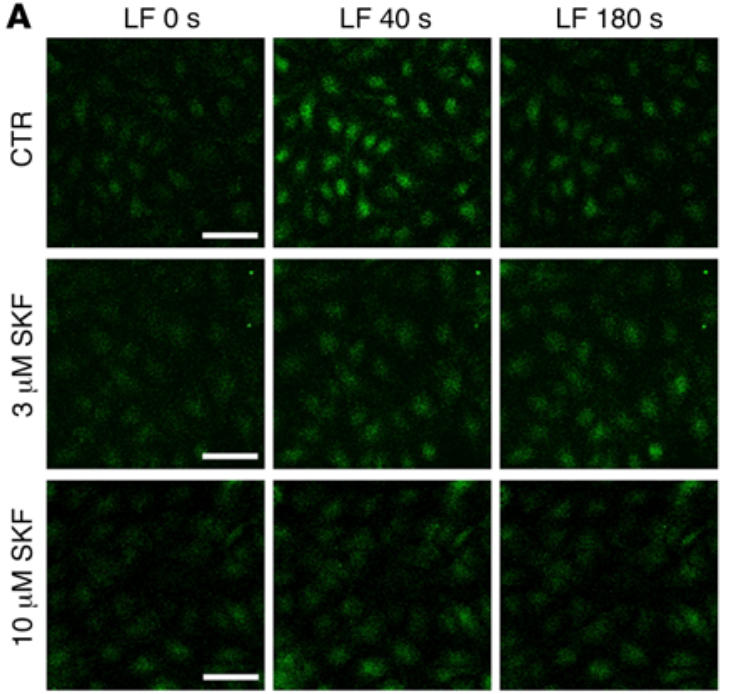

B

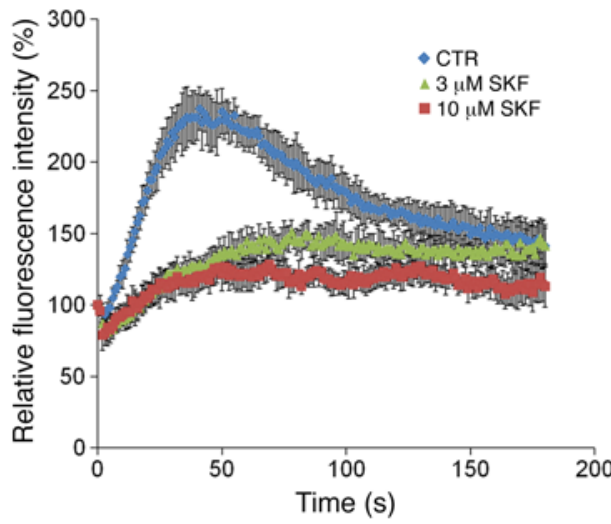

C
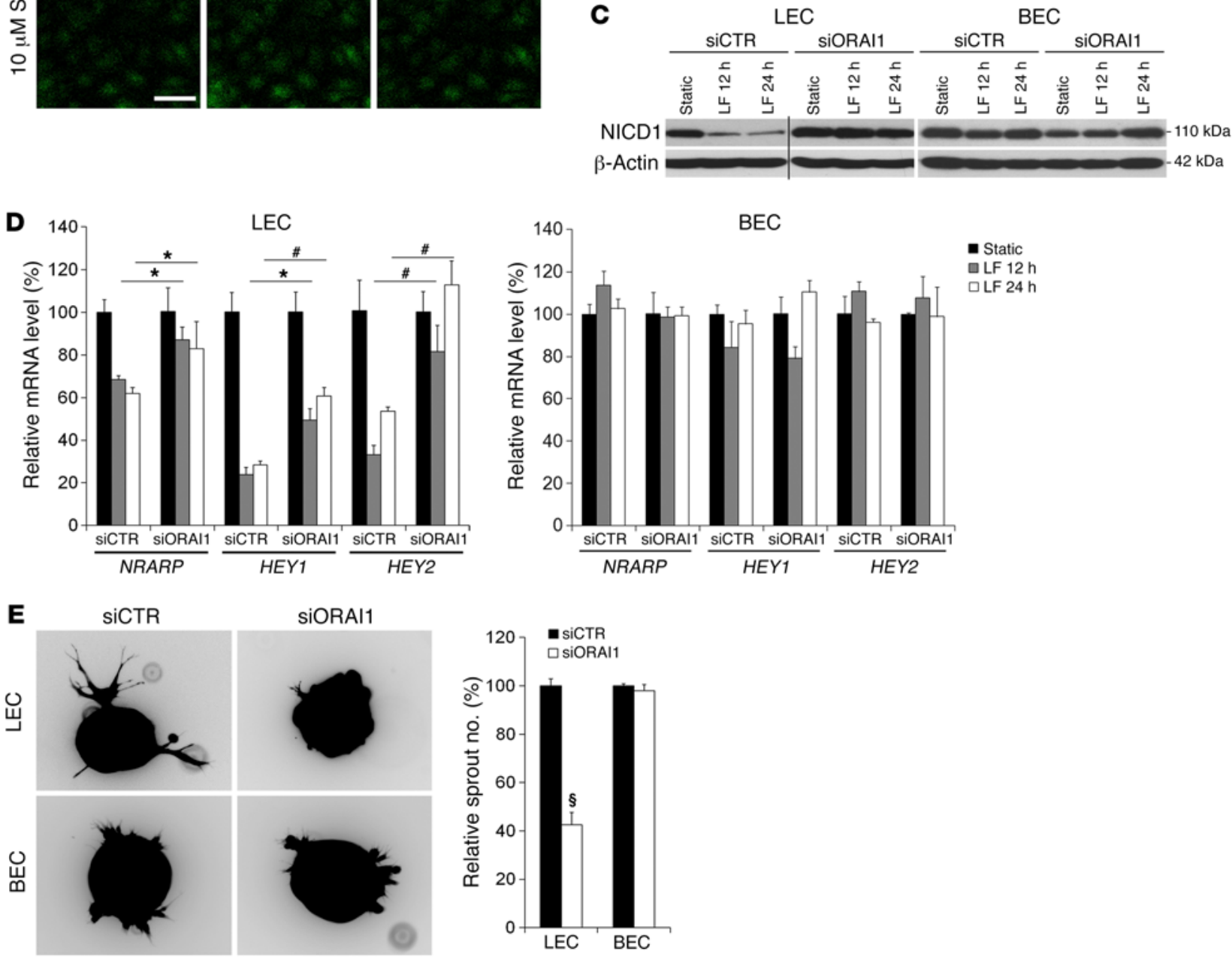

Figure 2. ORAI1 is essential for the laminar flow-induced NOTCH1 suppression in LECs. (A and B) Activation of Ca ${ }^{2+}$ mobilization in LECs by laminar flow: Fluo-4-loaded LECs were pretreated with PBS (CTR) or SKF-96365 (SKF), an inhibitor of store-operated Ca ${ }^{2+}$ entry (SOCE), for 10 minutes, and subjected to laminar flow $\left(2 \mathrm{dyn} / \mathrm{cm}^{2}\right)$. Calcium signals were captured by a time-lapse microscope (A). Relative signal intensity was plotted in the graph (B). (C) NICD1 protein level in LECs and BECs that were transfected with control siRNA (siCTR) or ORAl1 siRNA (siORAl1) for 24 hours, then subjected to laminar flow (2 dyn $\left./ \mathrm{cm}^{2}\right)$. The vertical line marks a boundary between 2 different areas in the same gel. Blots presented are derived from replicate samples run on parallel gels. (D) qRT-PCR data showing the mRNA level of NRARP, HEY1, and HEY2 in ORAl1-inhibited LECs and BECs. Statistical comparison was made between control siRNA and ORAl1 siRNA samples at each time point to assess the reversal of the laminar flow-mediated suppression of the genes. (E) Effect of ORAI1 knockdown on the flow-induced sprouting of LECs and BECs. Cells were transfected with control or ORAl1 siRNA for 24 hours, exposed to laminar flow $\left(2 \mathrm{dyn} / \mathrm{cm}^{2}\right)$ for 24 hours, stained with a CellTracker dye, aggregated in methylcellulose polymers, and embedded in Matrigel. After 24 hours, spheroid images (>20) were taken and relative sprout numbers were quantified by NIH ImageJ. Scale bars: $50 \mu \mathrm{m}$. Data are expressed as SEM and SD of a representative of 3 independent experiments. ${ }^{*} P<0.05 ;{ }^{\#} P<0.01 ;{ }^{\$} P<0.001 ; t$ test. 
(1.5- to 2-fold) between the branching points in the lymphatic networks. In contrast, blood vessels in Orai1 $\mathrm{KO}$ embryos and adults exhibited slightly denser vascular networks with increased number of branching points and marginally reduced distance between the branching points. These vascular patterning phenotypes were also observed in endothelial-specific, inducible Orai1 KO embryos

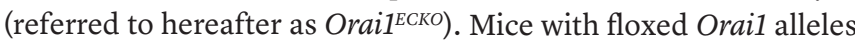
(Oraif $^{t / f l}$ ) were crossed with Prox1-EGFP lymphatic reporter mice (32) and Cdh5(PAC)-CreER ${ }^{T 2}$ (also known as VE-Cadherin-Cre$E R^{T 2}$ ) mice (33) expressing the tamoxifen-responsive Cre in endothelial cells. Orai1 ${ }^{E C K O}$ was induced by tamoxifen injection into pregnant female mice at E11.5 and E13.5. At E15.5, dermal lymphatics of Orai1 ${ }^{E C K O}$ embryos revealed a reduced number of lymphatic sprouts, and round/dull lymphatic tips that appeared less invasive. WT embryos, by comparison, showed growing lymphatic tips with jagged and sharp ends (Figure 3, C and E). Dermal blood vessels in Orai1 ${ }^{\text {EКО }}$ embryos displayed a higher vascular density than that of WT embryos (Figure 3, D and E). Taking these results together, genetic ablation of Orail results in decreased lymphatic sprouting in animal models, suggesting the essential role of Orail in lymphatic development.

Calcium signal activates CaM to interact with PROX1. We next investigated how the flow-induced calcium influx through ORAI1 causes the LEC-specific shear stress phenotypes, in particular NICD1 downregulation. Seeking initial insights into this question, we asked what LEC-specific molecules could be affected in their functions by calcium signaling. Interestingly, our bioinformaticsbased screening discovered that PROX1, the master regulator of lymphatic differentiation and development (34), contains a highprobability CaM-binding site in its $\mathrm{N}$-terminal region (Supplemental Figure 4A) and suggested a possible physical interaction between PROX1 and CaM proteins. We therefore generated an array of recombinant GST-PROX1 fusion fragments (Supplemental Figure 4B), and performed the CaM overlay assays (35) to evaluate the protein/protein interaction between PROX1 fragments and CaM. Indeed, the PROX1 D1 fragment, spanning from the first to the 168th amino acid of PROX1, displayed a strong binding affinity to $\mathrm{CaM}$ in the presence of calcium, but this interaction was abolished by a calcium chelator, EGTA (Figure 4A). We generated a panel of PROX1 substitution mutants by replacing the nonpolar/ hydrophobic amino acid residues in the predicted CaM-binding region (aa 14-35) with a polar/charged residue, aspartic acid (Figure $4 \mathrm{~B})$. We performed the CaM overlay assay using these substitution mutant fragments, and found that the V23D and V27D mutations abolished the PROX1/CaM interaction (Figure 4C), indicating the essential role of Val23 and Val27 in the interaction between PROX1 and CaM.

To identify the PROX1 binding region in CaM protein, we performed high-resolution NMR spectroscopy on the interaction between the $\mathrm{Ca}^{2+} / \mathrm{CaM}$ protein and a PROX1 peptide (aa 15-35). Addition of the PROX1 peptide into ${ }^{15} \mathrm{~N}$-labeled CaM caused large chemical shift changes for signals from the $\mathrm{N}$-terminal domain (e.g., I27, T29, A57, and D64; slow exchange) in the ${ }^{1} \mathrm{H},{ }^{15} \mathrm{~N}$ heteronuclear single quantum coherence NMR spectra (Figure 4D). In comparison, much smaller chemical shift perturbations were seen for the C-terminal domain (e.g., I100, I130, and N137; fast exchange), indicating that $\mathrm{Ca}^{2+} / \mathrm{CaM}$ interacts with the PROX1 peptide mainly through its $\mathrm{N}$-terminal region. To further confirm these biochemical data, we performed a series of co-immunoprecipitation (co-IP) assays for FLAG-tagged PROX1 and HA-tagged CaM proteins that were coexpressed in HEK293 cells with or without altering effective calcium concentration. These assays revealed that PROX1 could be coprecipitated with CaM, and also that the PROX1/CaM interaction could be inhibited by Bapta-AM (calcium chelator), but promoted by ionomycin ( $\mathrm{Ca}^{2+}$ ionophore) and $\mathrm{KCl}\left(\mathrm{Ca}^{2+}\right.$ influx inducer) (Figure $4 \mathrm{E}$ and Supplemental Figure $5 \mathrm{~A})$. In addition, we also carried out co-IP assays for endogenous PROX1 and CaM proteins in LECs that were pre-exposed to laminar flow. This study found that the PROX1/CaM interaction was significantly increased ( 7 -fold) in LECs by steady laminar flow, and that the increased interaction could be significantly reversed by Bapta-AM ( 3.5-fold) (Figure 4F and Supplemental Figure 5B).

We next investigated the impact of CaM overexpression on lymphatic sprouting using the GCaMP3 calcium reporter mouse. GCaMP3, a fusion protein of CaM and GFP, was originally developed as a calcium reporter. The GCaMP3 reporter mouse has been widely used as an animal model for live imaging of in vivo calcium dynamics (36). However, modifying its original purpose, we used the GCaMP3 reporter mouse as a transgenic model that conditionally overexpresses $\mathrm{CaM}$ in order to study the impact of overly expressed CaM protein on lymphangiogenesis. We first confirmed that PROX1 interacts with GCaMP3 through the CaM sequences (Supplemental Figure 6). Indeed, when GCaMP3 was conditionally overexpressed in Tie2- and Prox1-expressing cells during embryogenesis using Tie2-Cre and Prox1-CreER ${ }^{T 2}$, respectively, GCaMP3 overexpression unexpectedly caused extremely enlarged, abnormal lymphatic vessels with severely reduced branches (Figure 4G). We speculate that these surprising gain-offunction lymphatic phenotypes may be caused by disturbed calcium signaling due to an excessive expression of GCaMP3 in LECs. In summary, PROX1 physically interacts with CaM in a calciumdependent manner, and the PROX1/CaM interaction is dramatically enhanced by the steady laminar flow. Furthermore, ectopic overexpression of CaM causes abnormally enlarged lymphatic vessels with defective branching morphogenesis.

CaM promotes a complex formation between PROX1 and KLF2 in a calcium-dependent manner. From our separate effort to find PROX1-interacting proteins, we fortuitously identified KLF2 as a PROX1 binding protein (Figure 5A). Because KLF2 is the master regulator for shear stress responses (37) and is dramatically upregulated by steady laminar flow in LECs (Figure 1A), we asked whether KLF2 may be involved in the flow-induced Notch downregulation. Supporting this notion, the PROX1/KLF2 interaction was decreased by calcium chelation, but enhanced by ionomycin, in HEK293T cells (Figure 5B and Supplemental Figure 5C), indicating the dependence of the PROX1/KLF2 interaction on the presence of calcium. Moreover, the PROX1/KLF2 interaction was enhanced by ectopic expression of HA-tagged CaM (Figure 5C and Supplemental Figure 5D). Although endogenous CaM proteins may be abundantly present in the cells, an additional expression of CaM could further enhance the PROX1/KLF2 interaction.

These data raised a possibility that CaM may facilitate the interaction between PROX1 and KLF2 and form a triple protein complex in the presence of increased intracellular calcium. In 
A
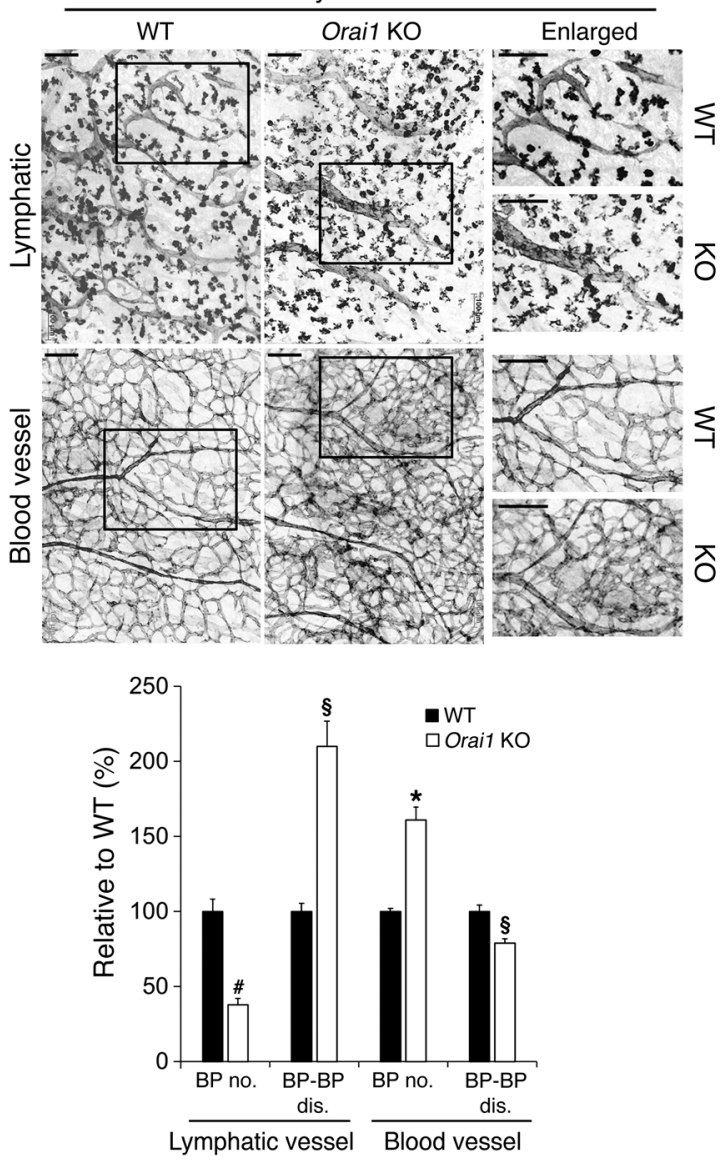

B
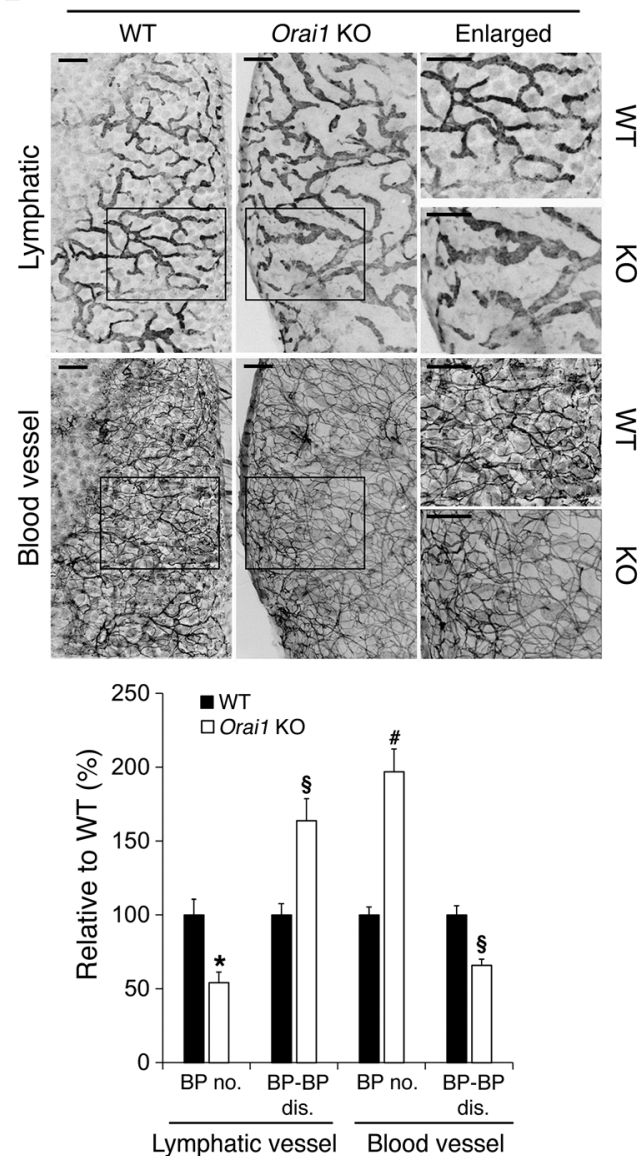

C

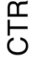

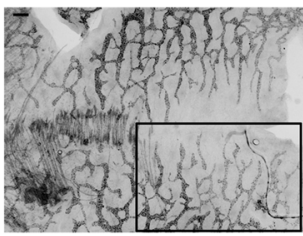

$\frac{0}{\frac{0}{4}}$
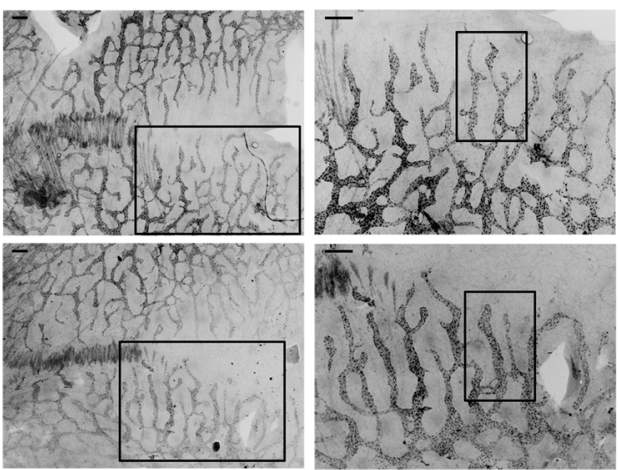

D

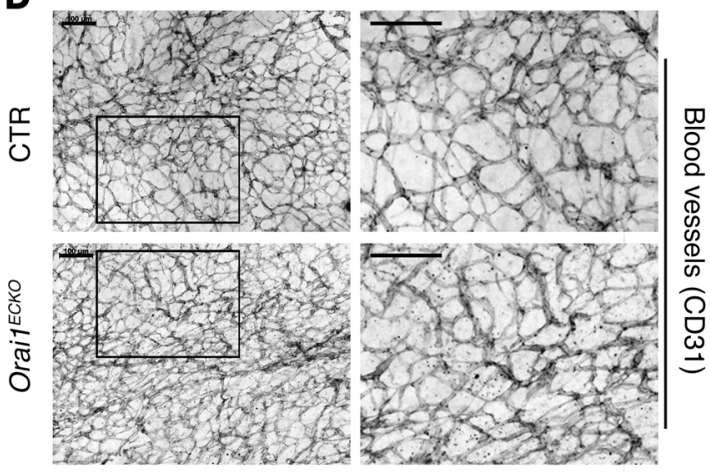

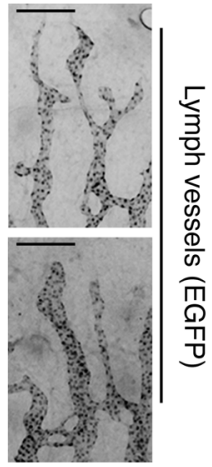

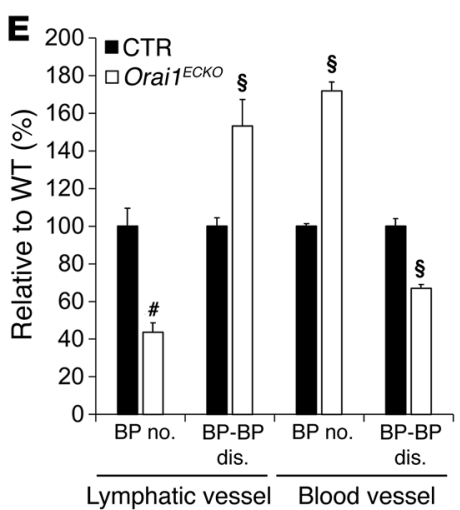

Figure 3. ORAl1 is required for normal lymphatic sprouting during development. (A) Immunofluorescence images of lymphatic and blood vessels in the back skins of WT and Orai1 KO embryos (E15.5). Lymphatics were stained for LYVE-1, and blood vessels were visualized by anti-CD31 staining. The graph shows the relative number of branching points (BP no.) and average distance between the branching points (BP-BP dis.). Scale bars: 100 $\mu \mathrm{m}$. (B) The ear vasculature of 3-week-old WT and Orai1 KO mice was similarly visualized and analyzed. Scale bars: $250 \mu \mathrm{m}$. (C-E) Endothelial-specific deletion of Orai1 (Orait ${ }^{\text {EKO }}$ ) was induced in pregnant females by peritoneal injection of tamoxifen $(1.5 \mathrm{mg})$ at E11.5 and 13.5. At E15.5, the embryos were harvested and genotyped. Lymphatics and blood vessels of the back skins of the control (CTR) embryos [Orai1/+C Cdh5(Pac)-CreER ${ }^{\text {T2 }}$ Prox1-EGFP] and Orai7 ${ }^{E C K O}$ embryos [Orai7 ${ }^{f / f l}$ Cdh5(Pac)-CreER ${ }^{\text {T2 }}$ Prox1-EGFP] were visualized by lymphaticspecific EGFP signals (C) and anti-CD31 staining (D), respectively. Scale bars: $250 \mu \mathrm{m}$ (C), 100 $\mu \mathrm{m}$ (D). (E) Vascular analyses were performed as described above. For all studies, a total of more than 6 embryos or postnatal mice per genotype collected from at least 3 independent litters were analyzed. The dorsal midline areas of the embryos were chosen as the sites for vascular analyses. Data are expressed as SEM and SD. ${ }^{*} P<0.05 ;{ }^{\#} P<0.01 ; \$ P<0.001$. 
A

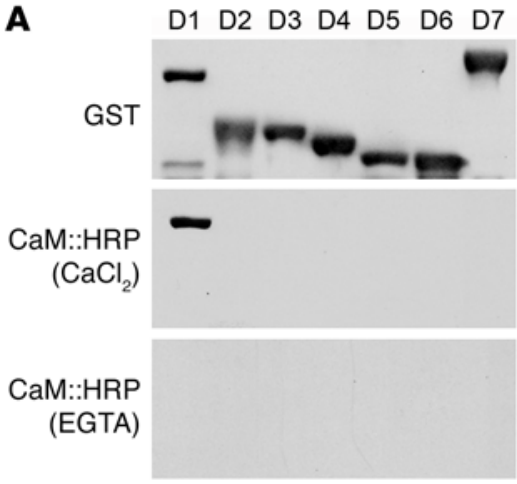

B 14-KRRRVDIGVKRTVGTÁ $\stackrel{+++}{+} \ddot{F}_{-3}$ V19D I21D V23D V27D

A30D

A32D

F33D

F34D
D

D D

D

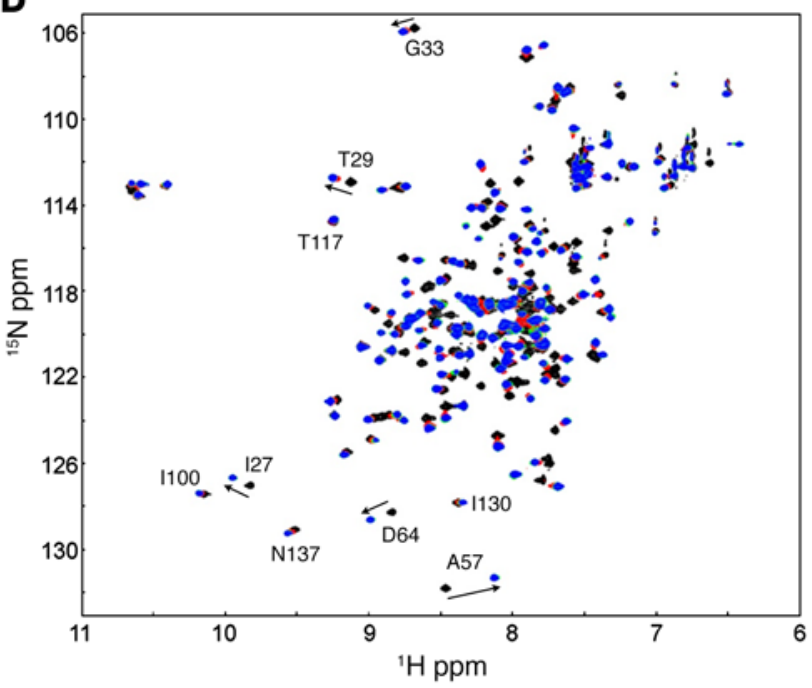

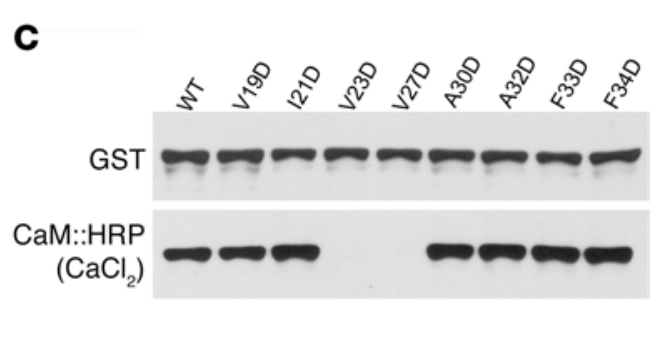

E

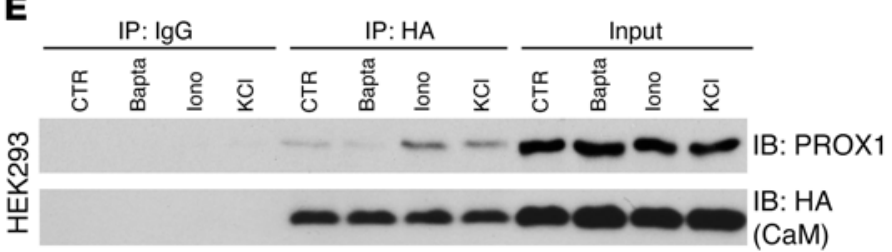

F

C

D

${ }_{D}$<smiles>[2H][10BH]</smiles>

G

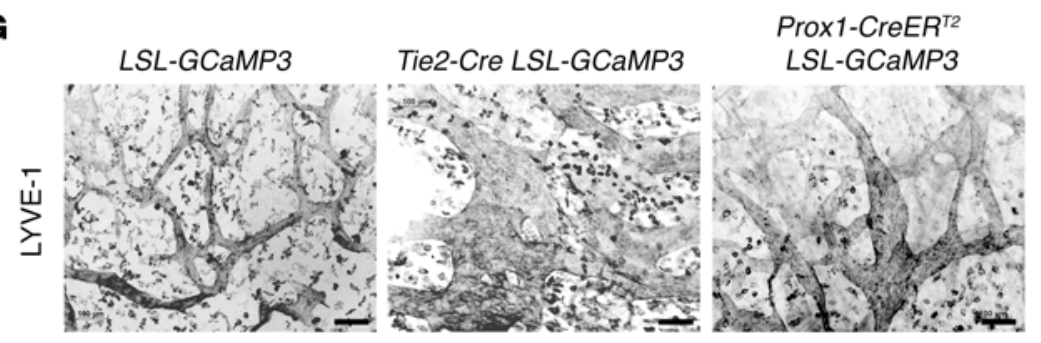

Figure 4. CaM physically interacts with PROX1 and regulates lymphatic sprouting. (A) CaM overlay assays. Top: Western analysis showing the quantity and quality of recombinant GST-PROX1 fragments (D1-D7; Supplemental Figure 4B). Middle and bottom: CaM overlay assays were performed on duplicate blots using the CaM::HRP conjugate protein in the presence of $\mathrm{CaCl}_{2}(1 \mathrm{mM}$, middle) or EGTA (5 mM, bottom). (B) Substitution mutations in the predicted CaM-binding site of PROX1: uncharged residues (V19, I21, V23, V27, A30, A32, F33, and F34) were replaced with aspartic acid, D. (C) CaM overlay assays performed against the PROX1 mutant fragments identified 2 residues (V23, V27) as essential for the CaM/PROX1 binding. (D) Superposed ${ }^{1} \mathrm{H},{ }^{15} \mathrm{~N}$ heteronuclear single quantum coherence spectra of Ca ${ }^{2+}$-saturated CaM recorded with 0 (black), 0.5 (red), 1.0 (green), and 1.5 (blue) molar equivalents of PROX1 peptide (aa 15-35). The assignments for separated signals are shown. Signals from the N-domain of CaM were perturbed in slow exchange on the NMR timescale, whereas the perturbations of the signals from the C-domain were relatively small and in fast exchange. (E) Co-IP assay demonstrating calciumdependent interaction of PROX1 and CaM. HEK293 cells were transfected with vectors expressing FLAG-tagged PROX1 and/or HA-tagged CaM, treated with Bapta-AM (Bapta, $3 \mu \mathrm{M}$ ), ionomycin (Iono, $1 \mu \mathrm{M})$, and $\mathrm{KCl}(40 \mathrm{mM})$, and co-IP was performed. Band intensity is quantitated in Supplemental Figure 5A. (F) Co-IP assay demonstrating that laminar flow (LF, 2 dyne/cm²) enhanced the interaction between PROX1 and CaM in LECs. LECs were treated with Bapta-AM ( $3 \mu \mathrm{M}$, Bapta) for 10 minutes, followed by laminar flow for 4 hours. Two CaM blots from a short or long exposure are shown. Band intensity is quantitated in Supplemental Figure 5B. (G) Whole-mount LYVE-1 staining of the back skin of embryos (E15.5) having LSL-GCaMP3, Tie2-Cre LSL-GCaMP3, or Prox1-CreER ${ }^{T 2}$ LSL-CCaMP3 transgenes. Pregnant mice were injected i.p. with tamoxifen (1.5 mg) at E11.5 and E13.5, and the embryos of each genotype

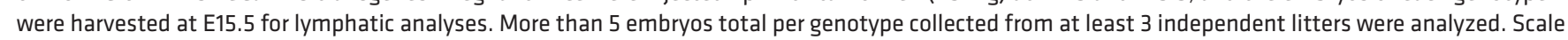
bars: $100 \mu \mathrm{m}$. For in vitro experiments, data are expressed as SEM and SD from a representative of 3 independent experiments. 

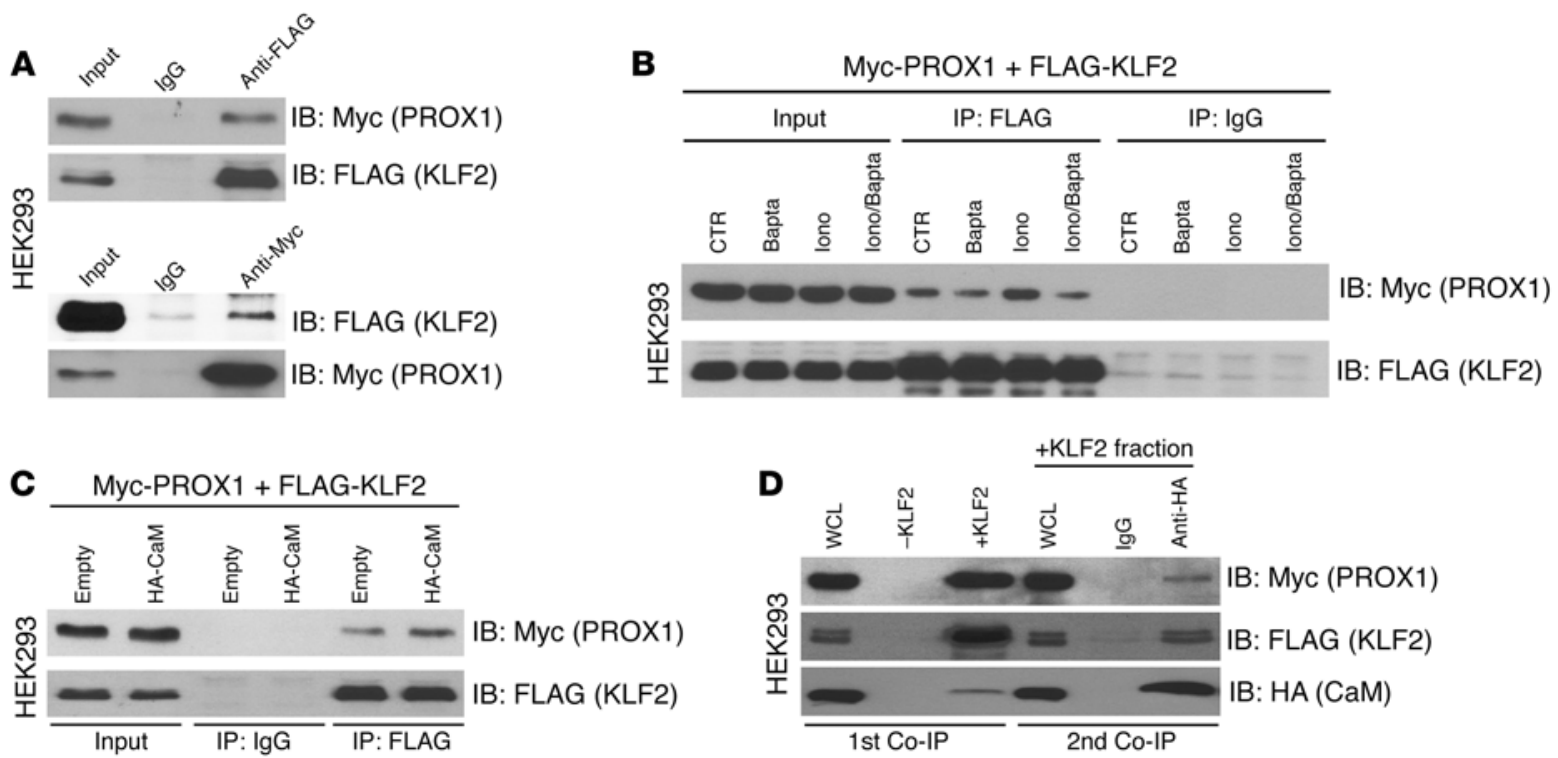

E

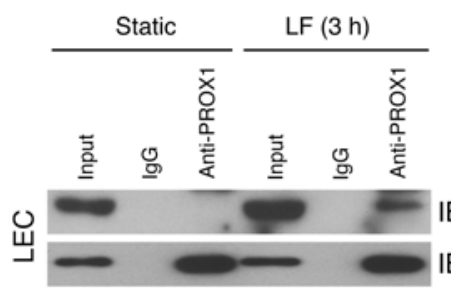

IB: KLF2

IB: PROX
$\mathbf{F}$

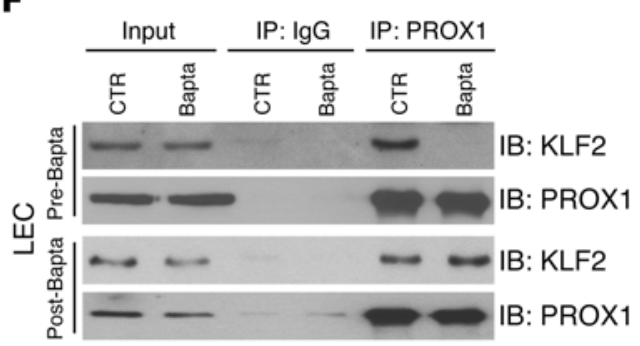

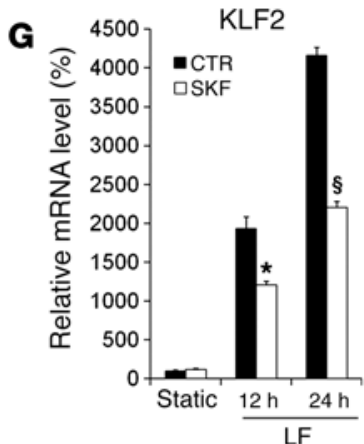

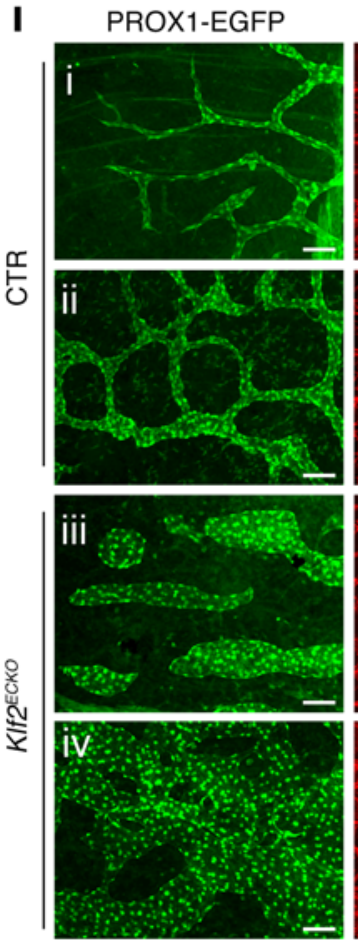

CD31
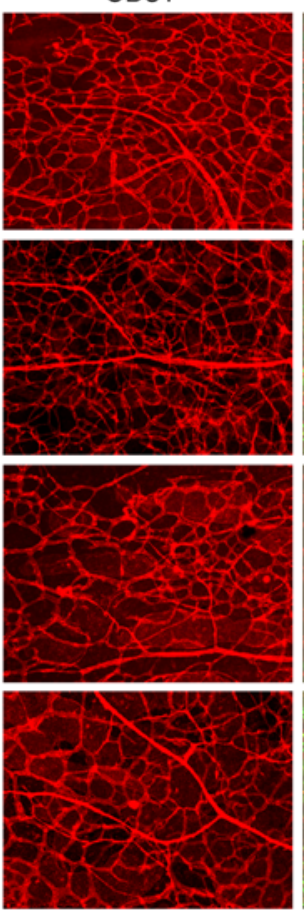

Merged
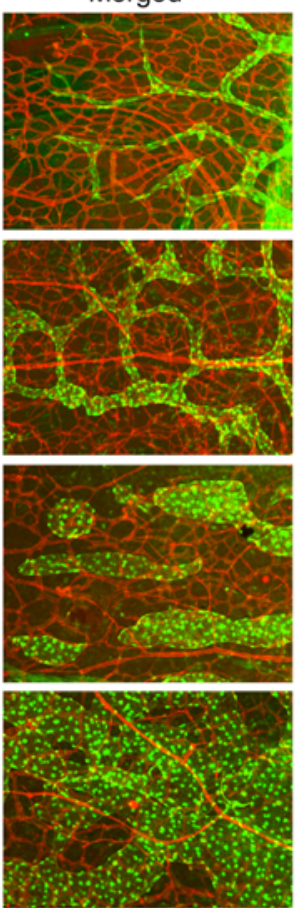
Figure 5. KLF2 forms a complex with PROX1 and CaM, and is required for lymphatic sprouting. (A) FLAG-KLF2 and/or Myc-PROX1 were expressed in HEK293 cells, and co-IP assays were performed using anti-FLAG (top panel) or anti-Myc antibody (bottom panel). (B) Co-IP assays were performed against FLAG-KLF2 and Myc-PROX1 in HEK293 cells in the presence of DMSO (CTR), Bapta-AM (Bapta, $10 \mu \mathrm{M}$ ), ionomycin (Iono, $1 \mu \mathrm{M}$ ), or Bapta-AM $(10 \mu \mathrm{M}) /$ ionomycin $(1 \mu \mathrm{M})$. (C) CaM promotes PROX1/KLF2 interaction. FLAG-KLF2 and Myc-PROX1 were expressed in HEK293 cells with CaM (HA-CaM) or not (Empty). Co-IP was performed using IgG or anti-FLAG antibody. (D) Serial Co-IP assays showing a protein complex formation among KLF2, PROX1, and CaM. These 3 proteins were expressed in HEK293 cells and first immunoprecipitated using FLAG-antibody beads (1st Co-IP) and then using anti-HA antibody (2nd Co-IP) for final immunodetection of Myc-PROX1. See details in Supplemental Information. (E) LECs were cultured under static or laminar flow $\left(2 \mathrm{dyn} / \mathrm{cm}^{2}\right)$, and co-IP assays were performed using IgC or anti-PROX1 antibody, followed by immunoblotting against KLF2 or PROX1. (F) Effect of calcium chelation on PROX1/KLF2 complex formation. (Pre-Bapta) LECs were pretreated with Bapta-AM (10 $\mu M)$ for 10 minutes and exposed to laminar flow $\left(2 \mathrm{dyn} / \mathrm{cm}^{2}\right)$ for 24 hours before co-IP assay. (Post-Bapta) LECs were exposed to laminar flow for 0.5 hours, treated with Bapta-AM $(10 \mu \mathrm{M})$, and subjected to laminar flow (2 $\mathrm{dyn} / \mathrm{cm}^{2}$ ) for an additional 0.5 hours before co-IP assay. (G) qRT-PCR analyses showing that ORAl1 inhibition by SKF-96365 (SKF, $3 \mu \mathrm{M}$ ) reduced the flow-induced KLF2 upregulation in LECs. (H) Western blot assays showing protein levels of KLF2, NICD1, and $\beta$-actin in LECs, which were transfected with scrambled siRNA (siCTR) or KLF2 siRNA (siKLF2) and exposed to laminar flow $\left(2 \mathrm{dyne} / \mathrm{cm}^{2}\right)$. A vertical line marks spliced lanes. (I and J) Lymphatic and blood vessels of the back skins of control (CTR) embryos [Cdh5(PAC)-CreER ${ }^{T 2}$ KIf2 ${ }^{+/+}$Prox1-EGFP] and KIf2 ${ }^{E C K O}$ embryos [Cdh5(PAC)CreER ${ }^{T 2} \mathrm{KIf}^{\mathrm{fl} / f l}$ Prox1-EGFP] were visualized by lymphatic-specific EGFP signal and CD31 immunostaining, respectively, at E15.5. Equivalent anatomic locations were chosen for i and iii, and for ii and iv. Scale bars: $100 \mu \mathrm{m}$. (J) Vascular analyses were performed and graphed. Data are expressed as SEM and SD of a representative of 3 independent experiments. ${ }^{*} P<0.05$; ${ }^{\#} P<0.01 ; \$ P<0.001$.

order to address this hypothesis, we asked whether these 3 proteins can be found in a complex at the same time, and thus performed a serial co-IP assay. Myc-PROX1, FLAG-KLF2, and HACaM were expressed in HEK293 cells. A negative control was also prepared without FLAG-KLF2. We first set up an immunoprecipitation of the KLF2-containing complexes from both cell lysates. From the KLF2 precipitates obtained from the KLF2-expressing cells, we next performed an immunoprecipitation of CaM protein. In this serially precipitated CaM-containing protein complex, we checked the presence or absence of PROX1 protein by Western blotting for PROX1 (Figure 5D). Indeed, PROX1 protein was found in the protein complex that was immunoprecipitated sequentially by KLF2 and CaM, suggesting that the 3 proteins form a stable multiprotein complex. Notably, the PROX1/KLF2 interaction was undetectable in the statically cultured LECs, but was strongly enhanced in LECs that were pre-exposed to laminar flow for 3 hours (Figure 5E). Importantly, calcium was required for the initial formation, but not for the maintenance, of the PROX1/KLF2 complex by laminar flow, because pretreatment of LECs with Bapta-AM prior to the onset of fluid flow efficiently blocked the flow-induced formation of the complex, whereas treatment of LECs after laminar flow exposure did not disrupt the already formed complex (Figure 5F). In sum, these studies suggest that laminar flow activates KLF2, PROX1, and CaM proteins to form a multiprotein complex in LECs in a calciumdependent manner.
KLF2 is essential for flow-induced NICD1 downregulation and lymphatic sprouting. Moreover, chemical inhibition of ORAI1 significantly suppressed the laminar flow-induced KLF2 upregulation in LECs (Figure 5G). In turn, when the flow-induced KLF2 expression was inhibited by siRNA-mediated knockdown in LECs, the laminar flow could no longer decrease the NICD1 protein level, indicating the essential role of KLF2 in the flow-mediated downregulation of NOTCH1 (Figure 5H). We next studied the in vivo effect of KLF2 inhibition on lymphatic development by inducing endothelial-specific knockout of Klf2 ( $\left.K l f 2^{E C K O}\right)$. For this, we intercrossed Cdh5(PAC)-CreER $R^{T 2}$ mice (33), Klf $2^{f / f l}$ mice (38), and Prox1-EGFP mice (32), and then compared the developing vascular phenotypes between the control embryos [Cdh5(PAC)-CreER ${ }^{T 2} \mathrm{Klf}^{+/+}$Prox1-EGFP] and Klf2 ${ }^{\text {ECKO }}$ embryos [Cdh5(PAC)-CreER ${ }^{T 2} \mathrm{Klf2}^{f l / f l}$ Prox1-EGFP]. Indeed, the majority of $K l f 2^{\text {ЕКО }}$ embryos (E15.5, $n>6$ ) developed severe nuchal edema, often displaying blood-filled lymphatics (Supplemental Figure 7). $K l f 2^{\text {ECKO }}$ embryos displayed profoundly enlarged lymphatic vessels and impaired lymphatic sprouting, determined by a reduced number of branching points and an increased distance between the branching points, compared with WT controls (Figure 5, I and J, and Supplemental Figure 8). Together, our data show that Klf2 is required for the laminar flow to downregulate NICD1, and also that KLF2 ablation causes abnormally enlarged lymphatic vessels with defective lymphatic sprouting.

PROX1/KLF2 selectively suppresses Notch by upregulation of DTX1 and DTX3L in LECs. The finding of LEC-specific suppression of Notch activity directed us to hypothesize that there may be Notch activity modifiers acting selectively in LECs. We thus searched for putative modifiers that were differentially expressed in LECs and BECs under the laminar flow conditions. DTX1 and DTX3L were identified from our transcriptional profiling analyses as the candidates based on their previous association with Notch signaling as a heterodimeric E3 ligase (39-42). While DTX3L mRNA was upregulated specifically in LECs in response to laminar flow, DTX1 mRNA was commonly upregulated in LECs and BECs (Figure 6, A and B). This finding was also confirmed by the protein levels (Supplemental Figure 9). ChIP assays revealed that PROX1 and KLF2 proteins were physically associated with the promoters of the DTX1 and DTX $3 L$ genes, and that their promoter occupancies were significantly increased by laminar flow. Notably, these flow-increased promoter occupancies were reversed by an inhibitor of SOCE (SKF-96365) (Figure 6, C and D), indicating that the flow-induced bindings of PROX1 and KLF2 to the promoters of DTX1 and DTX $3 L$ are dependent on ORAI1 function. Functionally, DTX1 and DTX3L proteins were found to synergistically reduce the endogenous NICD1 level in HEK293 cells and LECs (Figure 6, E and F, and Supplemental Figure 10, A and B). Simultaneous knockdown of DTX1 and DTX3L showed a synergy in reversing the flow-mediated decrease of NICD1 level in LECs (Figure 6G and Supplemental Figure 10C). Moreover, the flowinduced increase in sprouting capacity was significantly reduced by knockdown of DTX1 or DTX3L in LECs (Figure 6H), indicating the critical role of DTX1 and DTX3L in the flow-mediated promotion of lymphatic sprouting.

Since DTX3L was uniquely upregulated in LECs after exposure to laminar flow, we generated Dtx $3 l \mathrm{KO}$ mice using Cas 9 tech- 

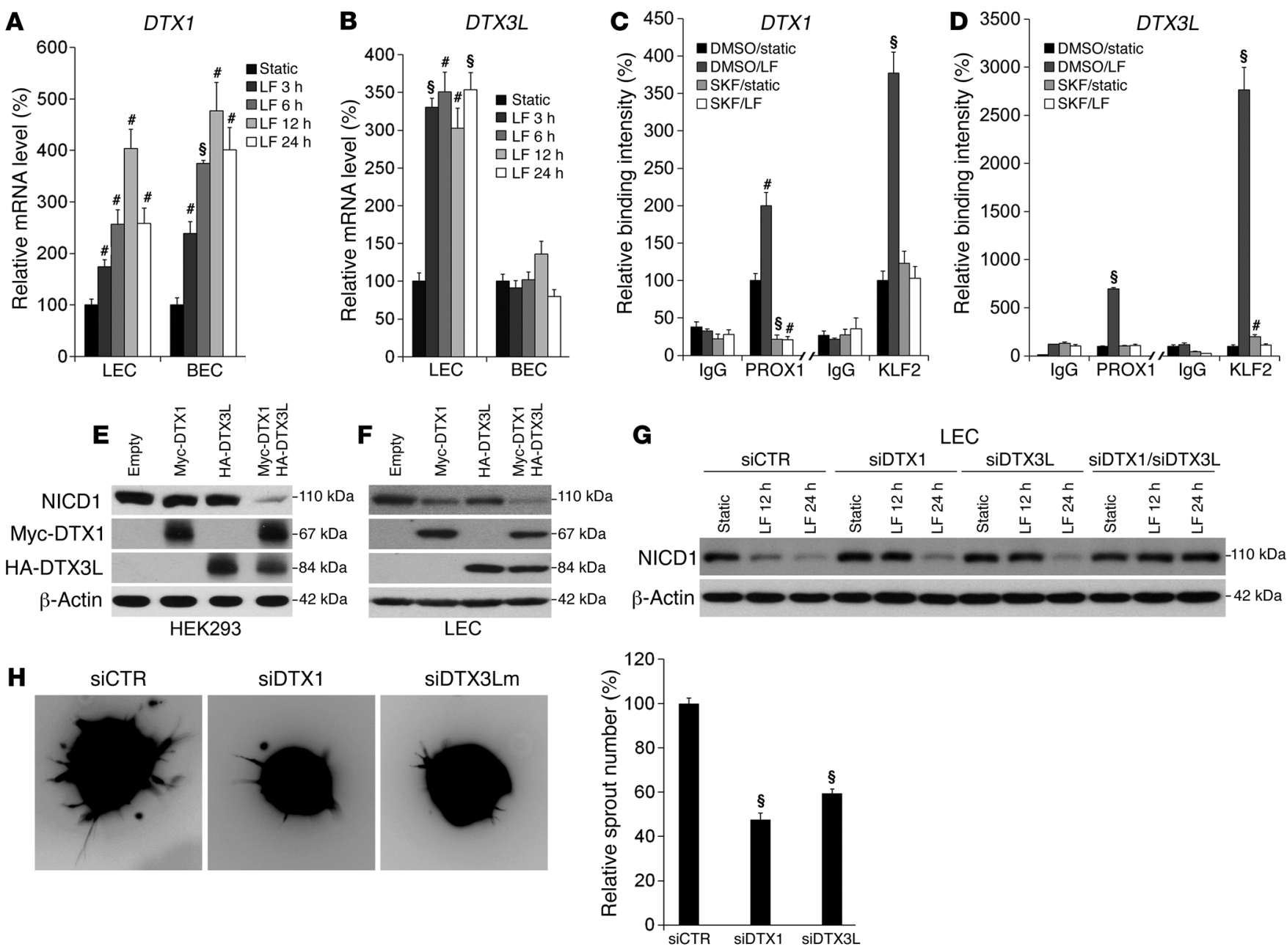

I

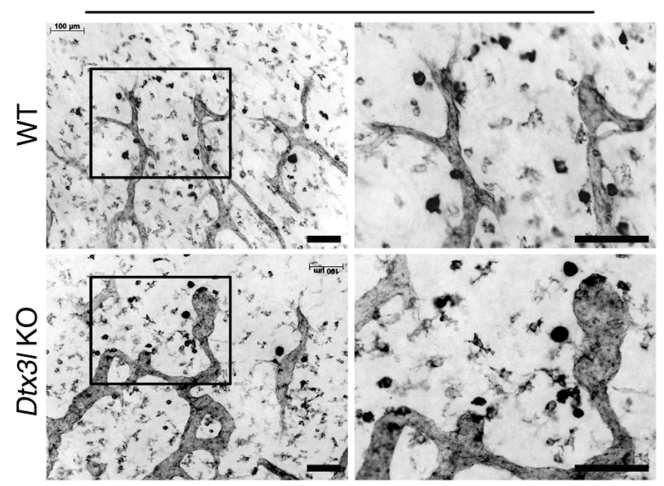

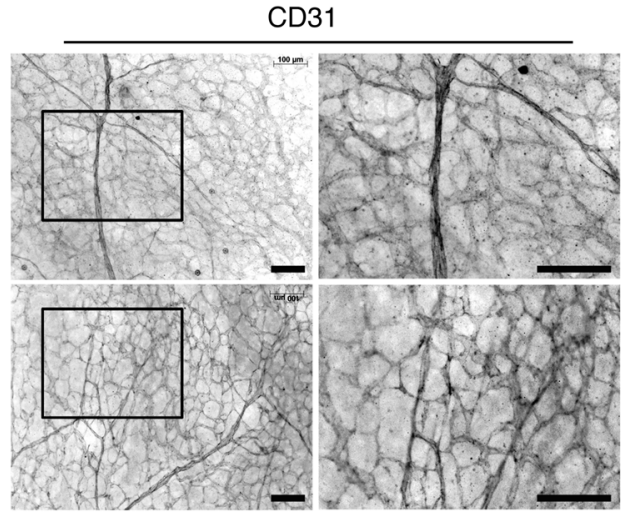

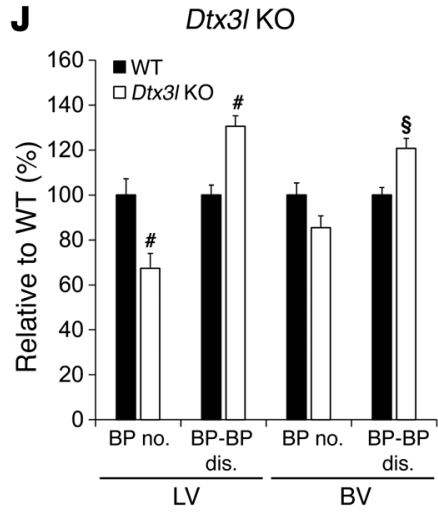

Figure 6. PROX1/KLF2 complex promotes lymphatic sprouting by upregulating DTX1 and DTX3L. (A and B) qRT-PCR analyses showing the expression of DTX1 (A) and DTX3L (B) in LECs and BECs exposed to laminar flow (2 dyne/cm²) for 0 (static), 3, 6, 12, and 24 hours. Protein expression is shown in Supplemental Figure 9. (C and D) ChIP assays demonstrating the binding of PROX1 and KLF2 to the promoters of the DTX1 (C) and DTX3L (D) genes in LECs, which were exposed to laminar flow for 0 (static) or 3 hours in the presence of vehicle (DMSO) or SKF-96365 (SKF, $3 \mu \mathrm{M}$ ). (E and F) Western blot assays showing a synergistic degradation of NICD1 by DTX1 and/or DTX3L in HEK293 cells (E) and LECs (F). Band intensities are graphed in Supplemental Figure 10, A and B. (C) Western blot assays showing the knockdown effect of DTX1 and/or DTX3L on the flow-induced downregulation of NICD1. LECs were transfected with siRNA for DTX1 and/or DTX3L for 24 hours, followed by laminar flow for 0 (static), 12, or 24 hours, and subjected to immunoblotting for NICD1 and $\beta$-actin. Band intensities were measured and are graphed in Supplemental Figure 10C. (H) Spheroid-based sprouting assay showing that sprouting capability was significantly reduced by knockdown of DTX1 or DTX3L. LECs were transfected with control siRNA (siCTR), siRNA for DTX1 (siDTX1), or siRNA for DTX3L (siDTX3L) for 12 hours, exposed to laminar flow $\left(2 \mathrm{dyn} / \mathrm{cm}^{2}\right)$ for 24 hours, and subjected to sprouting assays as described in Figure $1 E$ ( $n>20 \mathrm{spheroids)}$. (I) Lymphatics and blood vessels were visualized by LYVE-1 and CD31 staining, respectively, in the back skins of WT and Dtx3/ KO embryos (E15.5). Boxed areas are enlarged at right. Scale bars: $100 \mu \mathrm{m}$. (J) The number of branching points (BP no.) and distance between the branching points (BP-BP dis.) of lymphatic vessels (LV) and blood vessels (BV) were quantified and graphed. More than 4 embryos per genotype harvested from at least 3 independent litters were analyzed. Data are expressed as SEM and SD. ${ }^{\sharp} P<0.01 ;{ }^{\circledR} P<0.001$. 
nology (43) and studied the impact of genetic ablation of Dtx3l on embryonic lymphatic development. $D t x 3 l \mathrm{KO}$ mice apparently are fertile and grossly normal until 4-6 months of age, after which they tend to become unhealthy and die much earlier than their WT littermates. In developing embryos, DTX3L-deficient lymphatic vessels displayed defective lymphatic sprouting with a reduced number of branching points and an increased distance between the branching points, compared with those in WT embryos (Figure 6, I and J, and Supplemental Figure 11). In addition, whereas WT lymphatic vessels showed characteristic jagged and invasiveappearing tips, DTX3L-deficient lymphatic vessels had atypical dull and round vessel tips. Together, these results suggest that laminar flow upregulates a NOTCH1 E3 ligase, composed of DTX1 and DTX3L, which reduces NICD1 level in LECs and increases lymphatic sprouting.

ORAI1 and DTX $3 L$ are required for the suppression of Notch signal in developing lymphatic vessels. We next aimed to corroborate the epistatic relationships among ORAI1, KLF2, DTX3L, and Notch pathway, which were established based on the above in vitro experiments, using ORAI1 and DTX3L mutant embryos. For this purpose, we determined the mRNA levels of Klf2, Dtx3l, Nrarp, Hey1, and/or Hey2 in primary dermal LECs freshly isolated from the WT and mutant embryos. In LECs from Orai1 KO embryos, Klf2 and Dtx3l were found to be downregulated, while Nrarp, Hey1, and Hey 2 were upregulated, compared with those in LECs from the WT litter embryos (Figure 7A). Moreover, the Notch pathway genes were upregulated in LECs freshly isolated from Dtx3l KO embryos, compared with those in the WT LECs (Figure 7B). Consistent with these mRNA expression patterns, the protein levels of NICD1 were increased in the back skins and intestines of Orai1 KO (Figure 7C) and Dtx3l KO (Figure 7D) embryos. These data imply that ORAI1 and DTX3L are required for the suppression of Notch signal in embryonic LECs, presumably by functional fluid drainage during embryonic development.

DTX $3 L$ overexpression rescues lymphatic sprouting defects of Orai1 KO mouse. We next asked whether ectopic expression of DTX3L could rescue the defective lymphatic sprouting caused by genetic deletion of Orai1. For this, we generated an inducible Dtx3l transgenic mouse line, which harbors a transgene cassette consisting of transcription termination (poly-A) signals flanked by LoxP sites (LSL) that are followed by the Dtx3l-coding sequences ( $L S L-D t \times 3 l$ ). These LSL-Dtx3l transgenic mice were mated with Prox1-EGFP and Cdh5(PAC)-CreER $R^{T 2}$ mice, and resulting pregnant females were injected with tamoxifen at E11.5 and E13.5 for vascular analyses at E15.5 [LSL-Dtx3l Cdh5(PAC)-CreER ${ }^{T 2}$ Prox1-EGFP mice, hereafter known as $D t x 3 l^{T G}$ mice]. Indeed, endothelial-specific expression of $D t x 3 l$ in the transgenic $D t x 3 l^{T G}$ embryos caused a hypersprouting of the lymphatics, in comparison with the WT embryo (Figure 8, A and B). Interestingly, ectopic expression of Dtx3l also appeared to slightly increase blood vessel sprouting. We next intercrossed the LSL-Dtx3l Cdh5(PAC)-CreER ${ }^{T 2}$ mice (Dtx $3 l^{T G}$ mice without the Prox1-EGFP reporter) and/or Oraif ${ }^{l / f l}$ mice. The resulting pregnant females were injected with tamoxifen at E11.5 and 13.5, followed by embryo harvest at E15.5 for vascular analyses. As expected, Orai1 $^{E C K O}$ embryos [Orai1 ${ }^{f / f l} C d h 5(P A C)-C r e E R^{T 2}$ ] showed severely defective lymphatic sprouting (Figure 8, C and D). However, this defective lymphatic sprouting could be largely rescued by simul- taneous expression of exogenous Dtx3l in the Orai1 ${ }^{f l / f l} C d h 5(P A C)-$ CreER ${ }^{T 2} L S L-D t x 3 l$ (Orai1 ${ }^{E C K O} L S L-D t x 3 l$ ) mice, where the lymphatic sprouting and branching morphogenesis, determined by the number of branching points and the distance between the branching points, appeared to be comparable to those in the control embryos (Oral $^{f l / f l}$ and $\mathrm{Orai}^{+/ f l} L S L$-Dtx3l). Together, these data suggest that ORAI1 is essential for normal lymphatic sprouting by suppressing Notch activity via upregulation of DTX1 and DTX3L. Additionally, the defective lymphatic sprouting caused by Orail deletion could be rescued by ectopic expression of Dtx3l.

\section{Discussion}

Considering interstitial fluid drainage as the major function of the lymphatics, the basal-to-apical interstitial flow and the subsequent intraluminal flow caused by an overwhelming volume of tissue fluid could serve as an important expansion signal that triggers embryonic and postnatal lymphangiogenesis. This kind of fluid flow-driven lymphangiogenesis may be conceptually similar to hypoxia-activated angiogenesis in purpose to execute their physiological function. Fluid flow resulting from the functional drainage of embryonic fluid imposes shear stress on LECs in the lumen of developing lymphatic vessels. In this study, we investigated how laminar fluid flow activates lymphatic expansion during embryonic lymphatic development. Our in vitro and animalbased findings of this study allow us to build a working model for the molecular mechanism by which steady laminar flow induces lymphatic sprouting (Figure 8E). In our proposed model, steady laminar flow activates calcium influx in LECs through the ORAI1containing calcium channel and thus increases the concentration of calcium-loaded CaM. Increased $\mathrm{Ca}^{2+} / \mathrm{CaM}$, in turn, facilitates a complex formation between PROX1, CaM, and KLF2 proteins, which binds to the promoters of the DTX1 and DTX $3 L$ genes and activates their gene expression. Subsequently, upregulated DTX1 and DTX3L proteins form a heterodimeric NOTCH1 E3 ligase that degrades NICD1 protein, leading to activation of lymphatic sprouting. We propose that this mechanism, uniquely operating in lymphatic vessels, presents a key mechanotransduction pathway, through which LECs translate the hemodynamic force to a biological response, specifically, sprouting. In addition, we found in a separate study that steady laminar flow also enhances cell proliferation of LECs by upregulating VEGF-A, VEGF-C, and FGFR3 through the ORAI1/KLF2 pathway (44).

A previous study has nicely demonstrated the presence of embryonic fluid drainage and flow as early as mouse E11.5 and established the interrelationship among embryonic tissue fluid accumulation, fluid pressure increase, elongation and stretching of LECs, VEGFR-3 activation, LEC proliferation, and fluid drainage (6). Despite the convincing presence of lymphatic fluid flow at this developmental stage, however, the precise shear force level in developing embryonic lymphatic networks remains unknown and is thought to be experimentally challenging to measure. On the other hand, shear force levels in other postnatal lymphatics have been previously estimated. Under a basal physiological condition, the shear force level was approximately $0.64 \mathrm{dyn} / \mathrm{cm}^{2}$ in a collecting lymphatic vessel (45), $0.001 \mathrm{dyn} / \mathrm{cm}^{2}$ in mouse tail capillaries (46), and $0.003 \mathrm{dyn} / \mathrm{cm}^{2}$ in human skin capillaries (47). Compared with these very low shear force levels, however, conceptual predic- 
A

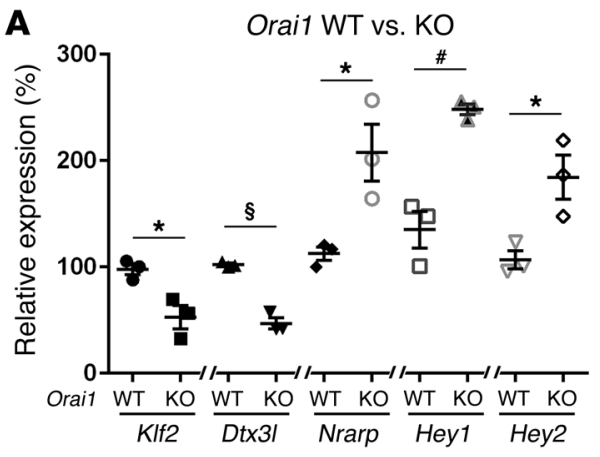

C

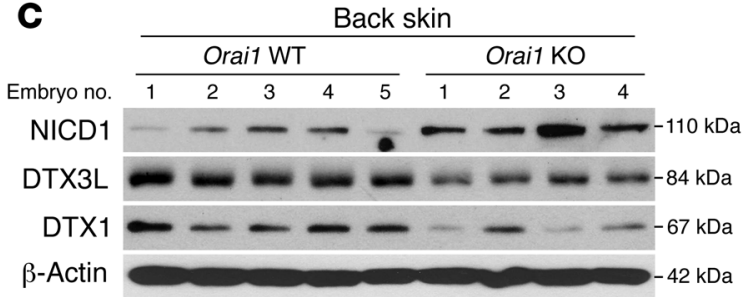

B

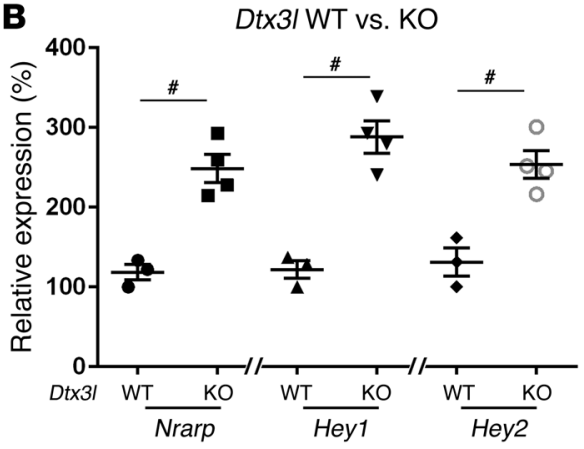

NICD1

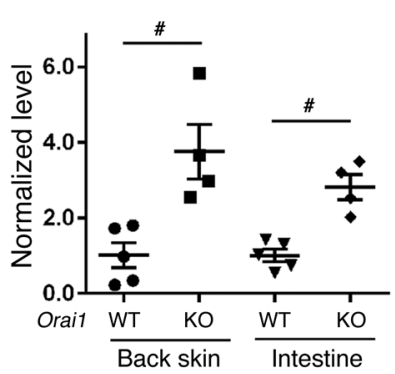

DTX3L

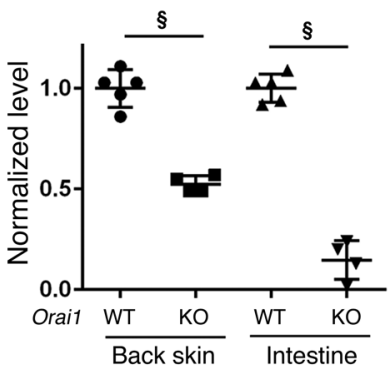

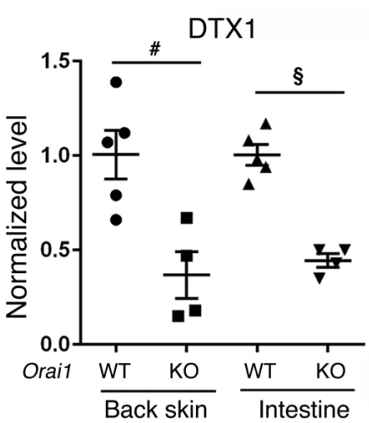

D Back skin
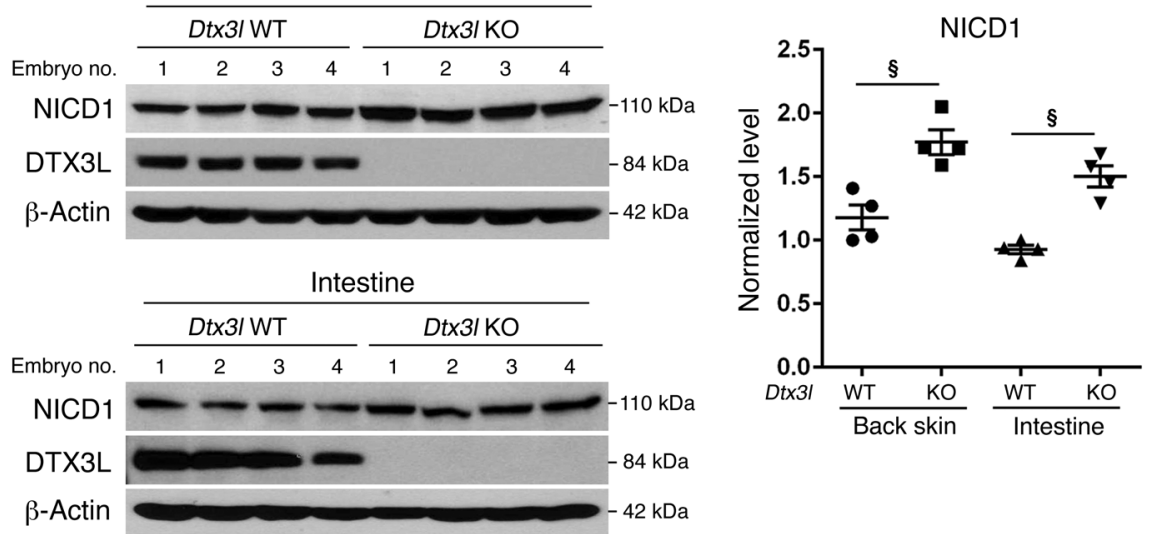

Figure 7. Regulation of Notch pathway genes by ORAl1 and DTX3L during lymphatic development. (A and B) qRT-PCR analyses showing the expression of KIf2, Dtx3l, Nrarp, Hey1, and/or Hey2 in dermal LECs freshly isolated from WT or KO embryos of Orai1 (A) or Dtx3l (B). (C and D) Western blot assays showing the protein expression of NICD1, DTX3L, and/or DTX1 in the back skins or intestines of WT versus mutant embryos of Orai1 (C) or Dtx3l (D). A monoclonal anti-NOTCH1 antibody that specifically detects the cleaved form of NOTCH1 at Val1744 was used to detect the NICD1 protein. Ratios of band intensities of NICD1, DTX3L, and DTX1 normalized against $\beta$-actin are graphed ( $n>4$ per genotype). Data are expressed as mean \pm SEM. Each data point represents an individual embryo $\left(n>3\right.$ per genotype). ${ }^{*} P<0.05 ;{ }^{*} P<0.01 ;{ }^{\circledR} P<0.001$.

tions would suggest that much higher levels of mechanical force may be required as growth stimuli to activate mature and quiescent postnatal lymphatic vessels to form new sprouts. Furthermore, developing lymphatics in rapidly growing embryos will experience much higher levels of hemodynamic force by dealing with an over- whelming amount of embryonic tissue fluid. To identify an optimal shear force condition, we have evaluated various force doses $\left(0.25-5 \mathrm{dyn} / \mathrm{cm}^{2}\right)$ on cultured human primary dermal LECs and found that a laminar shear force level higher than approximately $1 \mathrm{dyn} / \mathrm{cm}^{2}$ was needed to clearly trigger the classic endothelial 
A

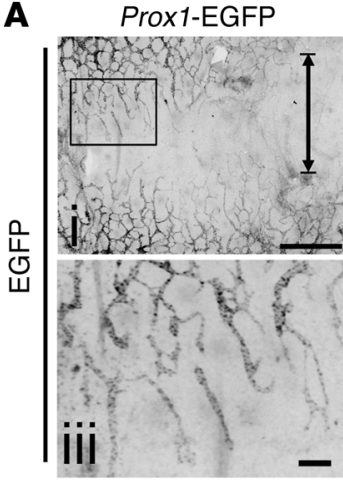

B

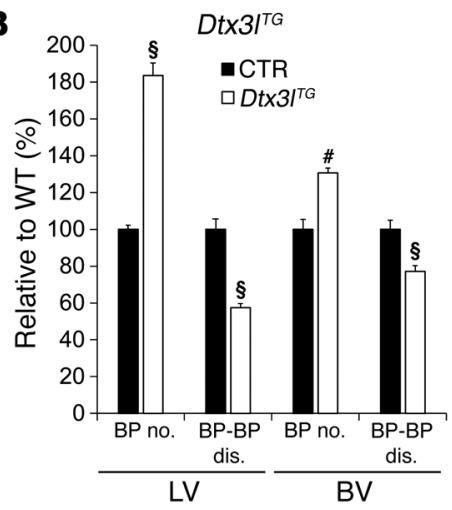

D

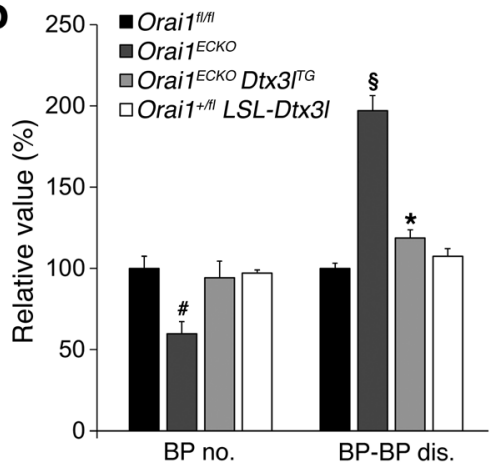

E

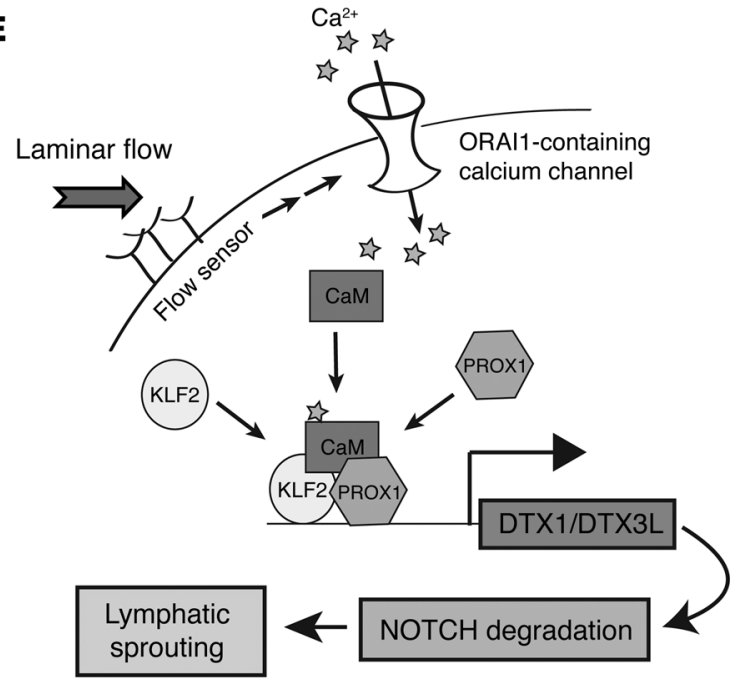

Prox1-EGFP

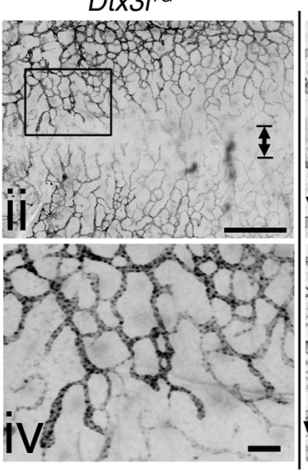

C
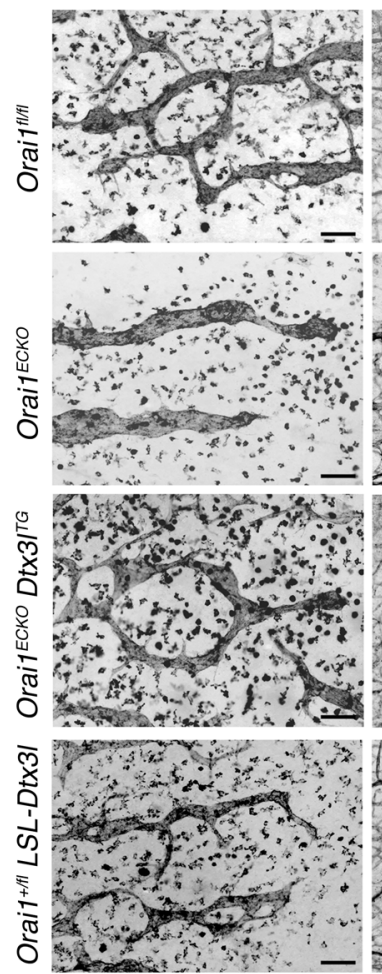
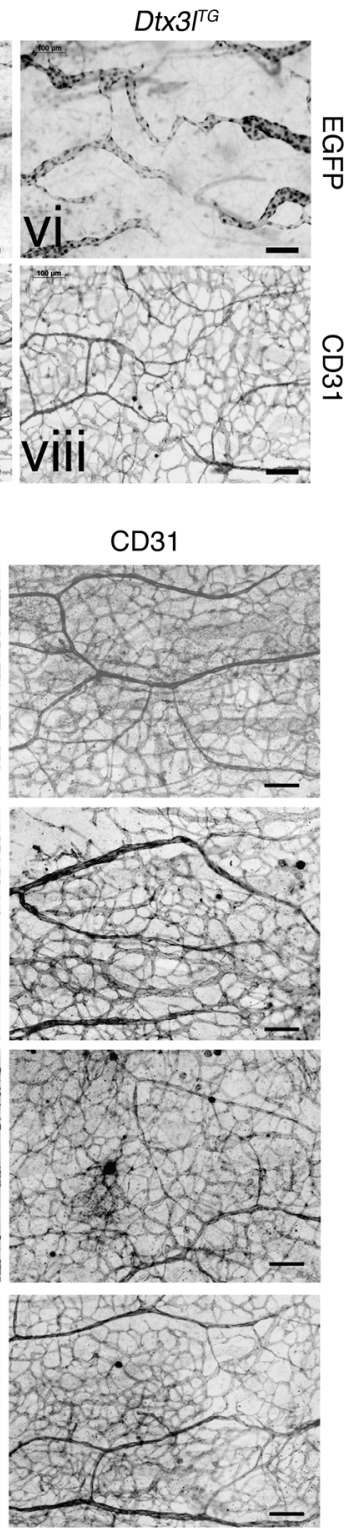

Figure 8. Overexpression of DTX3L rescues the defective lymphatic sprouting of Orai1 KO mice. (A and B) Lymphatic and blood vessels were visualized in control embryos (Prox 1 EGFP) or $D t \times 3 I^{T C}$ transgenic embryos

[Prox1-EGFP Cdh5(PAC)-CreER ${ }^{\text {T2 }}$

$L S L-D t \times 3 /]$. (A) Pregnant females were injected with tamoxifen at E11.5 and E13.5. At E15.5, dermal lymphatic and blood vessels were visualized by EGFP and CD31 staining, respectively. Panels $i$ and ii display lymphatic vessels growing into the dorsal midline region. Boxed areas are enlarged in panels iii and iv, respectively. Arrows indicate distance between front lines of lymphatic vessels growing from the lateral areas into the midline. Panels $v$ and vi highlight the dorsal midline area. Blood vessels in the corresponding area are shown in panels vii and viii, respectively. (B) Vascular analyses were performed and graphed to show branching point numbers (BP no.) and distance between the branching points (BPBP dis.) of lymphatic vessels (LV) and blood vessels (BV). More than 5 embryos total per genotype obtained from 3 independent litters were analyzed. The dorsal midline regions were imaged for the vascular analyses. (C and $\mathbf{D})$ Ectopic expression of DTX3L rescues the lymphatic sprouting defects in Orai7 $\mathrm{KO}$ embryos. (C) Endothelial-specific Orai7 ${ }^{\mathrm{CKO}}$ and/ or $D t x 3 /$ ectopic expression were induced in pregnant females at E11.5 and E13.5 by i.p. tamoxifen injection. Lymphatic and blood vessels were detected by LYVE-1 and CD31 staining, respectively, in the back skins of embryos with indicated genotypes at E15.5. (D) Vascular analyses were performed to determine the number of branching points (BP no.) and distance between the branching points (BP-BP dis.) of lymphatic vessels. (E) Schematic illustration showing a current working model of laminar flow-induced lymphatic sprouting. More than 4 embryos total per genotype were harvested from at least 3 independent litters and analyzed. The dorsal midline areas of the embryos were imaged for the vascular analyses. Scale bars: $100 \mu \mathrm{m}$ (A, iii-viii; C); 1 mm (A, i, ii). ${ }^{*} P<0.05 ;{ }^{*} P<0.01 ;{ }^{\circledR} P<0.001$. 
shear stress responses, such as cellular elongation, upregulation of KLF2 and endothelial NOS, and calcium influx (44), suggesting this range of fluid shear stress as a threshold shear force to trigger vascular remodeling phenotypes in vitro. In this study, we thus applied a shear force at approximately $2 \mathrm{dyn} / \mathrm{cm}^{2}$ to cultured LECs for all our experiments, except for the 3D biomimetic model (Figure $1 \mathrm{~F}$ and Supplemental Figure 1I), where approximately 5 $\mathrm{dyn} / \mathrm{cm}^{2}$ was applied to activate an efficient blood vessel growth. This minimum force level, similarly termed as a "set point" (48), for cultured LECs is consistent with an elegant study by Baeyens et al. (49), which demonstrated that the set point in LECs for the flow-induced cellular elongation and reorientation is much lower than that in HUVECs (10-20 dyn/ $\left./ \mathrm{cm}^{2}\right)$ because of the presence of VEGFR-3 in LECs. Moreover, separate studies have shown that flow-activated calcium influx in LECs reaches its maximum level at approximately $3 \mathrm{dyn} / \mathrm{cm}^{2}$ (28), whereas the half-maximal activation in HUVECs occurred at a shear stress of $30 \mathrm{dyn} / \mathrm{cm}^{2}(50)$.

LECs in developing, immature lymphatic vessels during embryogenesis appear to experience steady laminar flow, especially when the interstitial fluid load rapidly increases (6). Given the dynamics of embryonic interstitial fluid, we hypothesize that our working model for the laminar flow-induced lymphatic sprouting may be best applicable to early lymphatic capillaries during development, rather than more matured and large-caliber collecting lymphatics, where PROX1 expression is downregulated and oscillatory flow is the dominant pattern (51). It is important to note that the 2 flow patterns, laminar versus oscillatory, induce somewhat different shear stress transcriptional signatures in LECs. While laminar flow $\left(0.25-5 \mathrm{dyn} / \mathrm{cm}^{2}\right)$ profoundly induces KLF2 in LECs (Figure $1 \mathrm{~A})$, oscillatory flow $\left(\sim 4 \mathrm{dyn} / \mathrm{cm}^{2}\right)$ augments the valveforming cell characteristics in LECs without upregulation of KLF2 (8). Moreover, steady laminar flow condition did not change the expression of FOXC2, GATA2, and GJA4 (also known as CX37), which have been shown to be upregulated by oscillatory flow and to play key roles in lymphatic valve formation $(8,52-54)$. On the other hand, laminar flow was found to promote maturation of collecting lymphatic vessels during development (7), and laminar flow at the blood flow rate downregulates PROX1 and reprograms collecting lymphatics to adopt blood vessel phenotypes (18). Thus, fluid flow at different strengths and patterns may induce different responses in endothelial cells apparently through distinct mechanisms.

Notch signal plays a critical role in controlling vascular expansion and remodeling (19-23). Importantly, a reduction or loss of Notch signaling activates both blood vascular and lymphatic sprouting during development. Deltex (DTX) proteins were originally isolated as a regulator of Notch signaling in Drosophila. Deltex and its mammalian homolog DTX1 can either promote or inhibit Notch signaling depending on the cellular context. While deltex and DTX1 can promote Notch-dependent transcriptional activity, they also function as an E3 ligase that targets Notch protein for ubiquitination and degradation $(40,41,55,56)$. Notably, DTX3L interacts with DTX1 to form a more active heterodimeric E3 ligase $(39,42)$. Our study shows that DTX3L knockdown phenocopied ORAI1 knockdown in cultured LECs, abrogating flow-induced Notch downregulation (Figure 2C and Figure 6G). These in vitro data were further confirmed in animal models: Both Dtx3l knockout and Orai1 knockout increased NICD1 levels and upregulated the Notch target genes (Figure 7). An equally important finding is that both mRNA and protein levels of DTX3L were upregulated by laminar flow selectively in LECs but not in BECs (Figure 6B and Supplemental Figure 9). This implies that DTX3L is a critical player in the laminar flow-induced Notch downregulation in LECs. Supporting this notion, ectopic expression of DTX3L is sufficient to promote sprouting of both blood and lymphatic vessels (Figure 8, A and B). Together, a cooperative upregulation of DTX3L by PROX1, CaM, and KLF2 accounts for Notch downregulation in LECs and increased lymphatic sprouting in response to low-rate steady laminar flow.

Although both LECs and BECs display the classic responses to shear stress, only LECs downregulated Notch activity and enhanced sprouting, when exposed to low-rate steady laminar flow. In comparison, the same experimental condition did not trigger comparable phenotypes in BECs. It is necessary to exert caution in interpreting these data in BECs, because our shear force level was much lower than the physiological levels that BECs may experience in vivo. Nonetheless, Notch signal suppression through NICD1 downregulation may be a unique event that requires a lymphatic-specific mechanism. Indeed, NICD1 downregulation was found to be orchestrated by the lymphatic-specific protein PROX1 that interplays with the master regulator of the shear stress response, KLF2, and the $\mathrm{Ca}^{2+}$ signal mediator, CaM. Despite the clear presence of KLF2 upregulation and increased $\mathrm{Ca}^{2+}$ entry, laminar flow did not alter Notch activity in BECs, presumably because of the absence of PROX1. Therefore, we propose that the shear stress phenotype of BECs may be the "default" endothelial response, produced by KLF2 and CaM in the absence of the lymphatic-specific factor PROX1. In comparison, because BECs differentiate into LECs through the expression of PROX1, the unique shear stress phenotypes of LECs may be a "newly acquired" response choreographed by the lymphatic-specific PROX1 in collaboration with the 2 "old" players, KLF2 and CaM. Therefore, it would be very interesting to define how PROX1 can modify the collaborative function of KLF2 and CaM by comparing their functional interplays in LECs versus BECs. Moreover, it will be intriguing to define the molecular basis for the increased blood vessel network formation in Orai1 KO embryos.

In summary, fluid flow has long been thought to be a key nonbiological growth stimulus for lymphatic expansion. This is in agreement with the major function of the lymphatics, that is, draining interstitial fluid. Here, our working model illustrates a molecular mechanism underlying the lymphatic-specific activation of sprouting by laminar flow, underscoring the differential responses of the 2 vascular systems. Our study not only offers a better understanding of flow-induced lymphatic expansion, but also provides significant insight into other mechanotransduction pathways in endothelial cells.

\section{Methods}

Cell culture-related reagents and assays. Human primary endothelial cells (LECs and BECs) were cultured in media based on Endothelial Basal Media (EBM, Lonza) as previously described $(57,58)$. Primary cells under seventh population doublings (passage) were used for this study. HEK293 cells were obtained from American Type Culture Collection and cultured in DMEM (Genesee Scientific) supplemented 
with $10 \%$ calf serum and antibiotics (Invitrogen). Continuous laminar flow was generated based on the orbital rotation of culture medium over a monolayer of endothelial cells in a standard $\mathrm{CO}_{2}$ incubator as previously reported (15). Steady laminar flow was applied at $2 \mathrm{dyn} / \mathrm{cm}^{2}$ for all experiments in this study, except for Figure $1 \mathrm{~F}$ and Supplemental Figure 1I ( $\left.5 \mathrm{dyn} / \mathrm{cm}^{2}\right)$, as previously described (15). Spheroid-based sprouting assay was performed as described (25). Briefly, cells were exposed to laminar flow for 24 hours and then treated with a CellTracker (Molecular Probes; C7025, $1.5 \mu \mathrm{M}$ ) dye for 0.5 hours. If necessary, cells were transfected with siRNA for 24 hours before the onset of laminar flow. The cells were mixed with Methocel (20\%; Dow Chemical Co.), aggregated in hanging drops for 24 hours, and then embedded in Matrigel for 24 hours before imaging. Sources of antibodies for Western blot analyses and immunostaining are listed in Supplemental Table 1. See complete unedited blots in the supplemental material. The sources of reagents used in this study are: SKF-96365 hydrochloride, Tocris Bioscience; Tamoxifen Free Base, MP Biomedicals; and Bapta-AM, Sigma-Aldrich.

Animal-related studies. All mice were maintained and used for experiments in mixed backgrounds, except for Orail conventional KO mice, which were maintained in the C57BL/6 background. Sources of mice are: PROX1-EGFP, Jackson Laboratory [Tg(Prox1EGFP)KY221Gsat] (32); LSL-GCaMP3, Jackson Laboratory [B6;129SGt(ROSA)26Sortm38(CAG-GCaMP3)Hze/J]; Tie2-Cre, Jackson Laboratory; Cdh5(PAC)-CreER ${ }^{T 2}$, Ralf Adams, University of Münster (33); floxed $K l f 2\left(K l f 2^{f / f l}\right)$, Jerry Lingrel, University of Cincinnati (Cincinnati, Ohio, USA) (38); Prox1-CreER ${ }^{T 2}$, Taija Mäkinen, Uppsala University (Uppsala, Sweden) (52); and Orai1 $\mathrm{KO}(31,59)$ and floxed Orai1 (Orai1 ${ }^{f / f l}$ ), Yousang Gwack, UCLA. Dtx3l transgenic and KO mice were newly developed for this study in the University of Southern California Norris Comprehensive Cancer Center Transgenic/Knockout Rodent Core as described below. Orai1 ${ }^{l / f l}$ mice were generated in Yousang Gwack's laboratory by flanking of exon 2 of mouse Orail with LoxP sites by homologous recombination in AB2.2 (129SvEv) embryonic stem cells. Exon 2 encodes more than $50 \%$ of the protein, and its deletion results in loss of expression of Orai1. G418-resistant clones were screened by PCR for homologous recombination at both homology arms. Chimeric mice with floxed Orai1 alleles were generated by blastocyst injection of heterozygous $\mathrm{Oraif}^{\mathrm{ll}+}$ embryonic stem cell clones. Founder Orai $\mathrm{fl}^{\mathrm{fl+}}$ chimeric mice were bred with Flp-deleter mice (Jackson Laboratory) to remove the neomycin resistance gene cassette. Deletion of ORAI1 protein was validated by immunoblotting and measurement of SOCE. The $D t x 3 l$ conditional transgenic mouse line was generated by micro- injection of the gel-purified SaII/PacI fragment from the Dtx3l transgenic vector (YH3004; see below for detailed information). Dtx3l KO mice were generated by pronuclear injection of recombinant Cas 9 protein and 2 guide RNAs (AACUACUAAAAAGCCAGUAGAGG and UUGUGAUGACAUACAUCCCCAGG) for D $t x 3 l$ (PNA Bio) in the University of Southern California Norris Comprehensive Cancer Center Transgenic/Knockout Rodent Core.

Cellular, molecular, and biochemical assays. Detailed information on isolation of mouse embryonic dermal LECs, gene and protein expression, plasmids and vectors, mutagenesis, Notch activity reporter assay, confocal laser-scanning fluorescence microscopy for calcium imaging, CaM overlay assay, NMR study, co-IP, and 3D microfluidic sprouting assay is available in Supplemental Methods. Sequences of all the primers and siRNAs are listed in Supplemental Table 2.

Statistics. Student's $t$ test (2-tailed) was used to determine whether the differences between the experimental and control groups were statistically significant. A P value less than 0.05 was considered to be statistically significant. The analyses were performed using Microsoft Excel (Microsoft Office) and GraphPad Prism 6 (GraphPad Software Inc.).

Study approval. Human primary BECs and LECs were isolated and cultured from deidentified human neonatal foreskins with approval by the Institutional Review Board of the University of Southern California (principal investigator, YK Hong). Animal-related studies were approved by the University of Southern California Institutional Animal Care and Use Committee (principal investigator, YK Hong).

\section{Author contributions}

DC, EP, EJ, YJS, JY, EL, MH, SL, HI, and JB performed experiments and collected data. JPP, SS, YG, CSC, HV, AKW, and YKH designed and supervised the research. RHA and CJK provided the resources.

\section{Acknowledgments}

We thank Jerry Lingrel and Taija Mäkinen for sharing floxed Klf2 and Prox1-CreER ${ }^{T 2}$ mice, respectively. This study was supported by NIH grants (EY026260 to YKH, HL121036 to YKH, HL119583 to YKH, EB00262 to CSC, and EB017103 to CSC), an American Heart Association Grant-in-Aid (13GRNT17060131 to YKH), and the L.K. Whittier Foundation (to YKH and AKW).

Address correspondence to: Young-Kwon Hong, Department of Surgery, University of Southern California, Norris Comprehensive Cancer Center, 1450 Biggy Street NRT6501, Los Angeles, California 90033, USA. E-mail: young.hong@usc.edu.
1. Escobedo N, Oliver G. Lymphangiogenesis: origin, specification, and cell fate determination. Annu Rev Cell Dev Biol. 2016;32:677-691.

2. Swartz MA, Boardman KC. The role of interstitial stress in lymphatic function and lymphangiogenesis. Ann N Y Acad Sci. 2002;979:197-210.

3. Ng CP, Helm CL, Swartz MA. Interstitial flow differentially stimulates blood and lymphatic endothelial cell morphogenesis in vitro. Microvasc Res. 2004;68(3):258-264.

4. Boardman KC, Swartz MA. Interstitial flow as a guide for lymphangiogenesis. Circ Res. 2003;92(7):801-808.

5. Breslin JW, Kurtz KM. Lymphatic endothelial cells adapt their barrier function in response to changes in shear stress. Lymphat Res Biol. 2009;7(4):229-237.

6. Planas-Paz L, Strilić B, Goedecke A, Breier G, Fässler R, Lammert E. Mechanoinduction of lymph vessel expansion. EMBO J. 2012;31(4):788-804.

7. Sweet DT, et al. Lymph flow regulates collecting lymphatic vessel maturation in vivo. JClin Invest. 2015;125(8):2995-3007.

8. Sabine A, et al. Mechanotransduction, PROX1, and FOXC2 cooperate to control connexin 37 and calcineurin during lymphatic-valve formation. Dev Cell. 2012;22(2):430-445.
9. Kim S, Chung M, Jeon NL. Three-dimensional biomimetic model to reconstitute sprouting lymphangiogenesis in vitro. Biomaterials. 2016;78:115-128.

10. Schwartz MA, Simons M. Lymphatics thrive on stress: mechanical force in lymphatic development. EMBO J. 2012;31(4):781-782.

11. Masumura T, Yamamoto K, Shimizu N, Obi S, Ando J. Shear stress increases expression of the arterial endothelial marker ephrinB2 in murine ES cells via the VEGF-Notch signaling pathways. Arterioscler Thromb Vasc Biol. 2009;29(12):2125-2131.

12. Levesque MJ, Nerem RM, Sprague EA. Vascular endothelial cell proliferation in culture and the 
influence of flow. Biomaterials. 1990;11(9):702-707.

13. Akimoto S, Mitsumata M, Sasaguri T, Yoshida Y. Laminar shear stress inhibits vascular endothelial cell proliferation by inducing cyclin-dependent kinase inhibitor p21(Sdi1/Cip1/Waf1). Circ Res. 2000;86(2):185-190.

14. Lin K, et al. Molecular mechanism of endothelial growth arrest by laminar shear stress. Proc Natl Acad Sci U S A. 2000;97(17):9385-9389.

15. dela Paz NG, Walshe TE, Leach LL, Saint-Geniez M, D'Amore PA. Role of shear-stress-induced VEGF expression in endothelial cell survival. JCell Sci. 2012;125(pt 4):831-843.

16. Ando J, Yamamoto K. Vascular mechanobiology: endothelial cell responses to fluid shear stress. Circ J. 2009;73(11):1983-1992.

17. Jahnsen ED, Trindade A, Zaun HC, Lehoux S, Duarte A, Jones EA. Notch1 is pan-endothelial at the onset of flow and regulated by flow. PLoS One. 2015;10(4):e0122622.

18. Chen CY, et al. Blood flow reprograms lymphatic vessels to blood vessels. JClin Invest. 2012;122(6):2006-2017.

19. Zheng W, et al. Notch restricts lymphatic vessel sprouting induced by vascular endothelial growth factor. Blood. 2011;118(4):1154-1162.

20. Fatima A, et al. Murine Notch 1 is required for lymphatic vascular morphogenesis during development. Dev Dyn. 2014;243(7):957-964.

21. Niessen K, et al. The Notch1-Dll4 signaling pathway regulates mouse postnatal lymphatic development. Blood. 2011;118(7):1989-1997.

22. Domigan CK, Iruela-Arispe ML. Recent advances in vascular development. Curr Opin Hematol. 2012;19(3):176-183.

23. Roca C, Adams RH. Regulation of vascular morphogenesis by Notch signaling. Genes Dev. 2007;21(20):2511-2524.

24. Korff T, Krauss T, Augustin HG. Three-dimensional spheroidal culture of cytotrophoblast cells mimics the phenotype and differentiation of cytotrophoblasts from normal and preeclamptic pregnancies. Exp Cell Res. 2004;297(2):415-423.

25. Alajati A, et al. Spheroid-based engineering of a human vasculature in mice. Nat Methods. 2008;5(5):439-445.

26. Wong KH, Chan JM, Kamm RD, Tien J. Microfluidic models of vascular functions. Annu Rev Biomed Eng. 2012;14:205-230.

27. Galie PA, Nguyen DH, Choi CK, Cohen DM, Janmey PA, Chen CS. Fluid shear stress threshold regulates angiogenic sprouting. Proc Natl Acad Sci U S A. 2014;111(22):7968-7973.

28. Jafarnejad M, Cromer WE, Kaunas RR, Zhang SL, Zawieja DC, Moore JE. Measurement of shear stress-mediated intracellular calcium dynamics in human dermal lymphatic endothelial cells. Am J Physiol Heart Circ Physiol. 2015;308(7):H697-H706.
29. Singh A, Hildebrand ME, Garcia E, Snutch TP. The transient receptor potential channel antagonist SKF96365 is a potent blocker of low-voltageactivated T-type calcium channels. Br J Pharmacol. 2010;160(6):1464-1475.

30. Srikanth S, Gwack Y. Molecular regulation of the pore component of CRAC channels, Orai1. Curr Top Membr. 2013;71:181-207.

31. Gwack Y, et al. Hair loss and defective T- and B-cell function in mice lacking ORAI1. Mol Cell Biol. 2008;28(17):5209-5222.

32. Choi I, et al. Visualization of lymphatic vessels by Prox1-promoter directed GFP reporter in a bacterial artificial chromosome-based transgenic mouse. Blood. 2011;117(1):362-365.

33. Wang Y, et al. Ephrin-B2 controls VEGF-induced angiogenesis and lymphangiogenesis. Nature. 2010;465(7297):483-486.

34. Wigle JT, Oliver G. Prox1 function is required for the development of the murine lymphatic system. Cell. 1999;98(6):769-778.

35. Yoo JH, et al. Regulation of the dual specificity protein phosphatase, DsPTP1, through interactions with calmodulin. J Biol Chem. 2004;279(2):848-858.

36. Zariwala HA, et al. A Cre-dependent GCaMP3 reporter mouse for neuronal imaging in vivo. J Neurosci. 2012;32(9):3131-3141.

37. Lee JS, et al. Klf2 is an essential regulator of vascular hemodynamic forces in vivo. Dev Cell. 2006;11(6):845-857.

38. Wani MA, Means RT, Lingrel JB. Loss of LKLF function results in embryonic lethality in mice. Transgenic Res. 1998;7(4):229-238.

39. Yan $\mathrm{Q}$, et al. BBAP monoubiquitylates histone $\mathrm{H} 4$ at lysine 91 and selectively modulates the DNA damage response. Mol Cell. 2009;36(1):110-120.

40. Zhang P, Yang Y, Nolo R, Zweidler-McKay PA, Hughes DP. Regulation of NOTCH signaling by reciprocal inhibition of HES1 and Deltex 1 and its role in osteosarcoma invasiveness. Oncogene. 2010;29(20):2916-2926.

41. Mukherjee A, Veraksa A, Bauer A, Rosse C, Camonis J, Artavanis-Tsakonas S. Regulation of Notch signalling by non-visual beta-arrestin. Nat Cell Biol. 2005;7(12):1191-1201.

42. Takeyama K, et al. The BAL-binding protein BBAP and related Deltex family members exhibit ubiquitin-protein isopeptide ligase activity. J Biol Chem. 2003;278(24):21930-21937.

43. Shui B, Hernandez Matias L, Guo Y, Peng Y. The rise of CRISPR/Cas for genome editing in stem cells. Stem Cells Int. 2016;2016:8140168.

44. Choi D, et al. ORAI1 activates proliferation of lymphatic endothelial cells in response to laminar flow through Krüppel-like factors 2 and 4 [published online ahead of print February 6, 2017]. Circ Res. https://doi.org/10.1161/ CIRCRESAHA.116.309548.
45. Dixon JB, Greiner ST, Gashev AA, Cote GL, Moore JE, Zawieja DC. Lymph flow, shear stress, and lymphocyte velocity in rat mesenteric prenodal lymphatics. Microcirculation. 2006;13(7):597-610.

46. Berk DA, Swartz MA, Leu AJ, Jain RK. Transport in lymphatic capillaries. Am J Physiol. 1996;270(1 pt 2):H330-H337.

47. Fischer M, et al. Flow velocity of single lymphatic capillaries in human skin. Am J Physiol. 1996;270(1 pt 2):H358-H363.

48. Rodbard S. Vascular caliber. Cardiology. 1975;60(1):4-49.

49. Baeyens N, et al. Vascular remodeling is governed by a VEGFR3-dependent fluid shear stress set point. Elife. 2015;4:04645.

50. Schwarz G, Callewaert G, Droogmans G, Nilius B. Shear stress-induced calcium transients in endothelial cells from human umbilical cord veins. JPhysiol (Lond). 1992;458(1):527-538.

51. Norrmén C, et al. FOXC2 controls formation and maturation of lymphatic collecting vessels through cooperation with NFATc1. JCell Biol. 2009;185(3):439-457.

52. Bazigou E, et al. Genes regulating lymphangiogenesis control venous valve formation and maintenance in mice. JClin Invest. 2011;121(8):2984-2992.

53. Kazenwadel J, et al. GATA2 is required for lymphatic vessel valve development and maintenance. JClin Invest. 2015;125(8):2979-2994.

54. Kanady JD, Dellinger MT, Munger SJ, Witte MH, Simon AM. Connexin37 and Connexin 43 deficiencies in mice disrupt lymphatic valve development and result in lymphatic disorders including lymphedema and chylothorax. Dev Biol. 2011;354(2):253-266.

55 . Yamamoto N, et al. Role of Deltex-1 as a transcriptional regulator downstream of the Notch receptor. J Biol Chem. 2001;276(48):45031-45040.

56. Matsuno K, et al. Involvement of a proline-rich motif and RING-H2 finger of Deltex in the regulation of Notch signaling. Development. 2002;129(4):1049-1059.

57. Shin JW, et al. Prox1 promotes lineage-specific expression of fibroblast growth factor (FGF) receptor-3 in lymphatic endothelium: a role for FGF signaling in lymphangiogenesis. Mol Biol Cell. 2006;17(2):576-584.

58 . Lee $\mathrm{S}$, et al. Prox1 physically and functionally interacts with COUP-TFII to specify lymphatic endothelial cell fate. Blood. 2009;113(8):1856-1859.

59. Kim KD, Srikanth S, Yee MK, Mock DC, Lawson GW, Gwack Y. ORAI1 deficiency impairs activated T cell death and enhances T cell survival. Jimmunol. 2011;187(7):3620-3630.

60. Minoguchi S, et al. RBP-L, a transcription factor related to RBP-Jк. Mol Cell Biol. 1997;17(5):2679-2687. 